YanYan Li

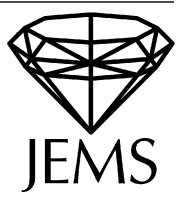

\title{
Remark on some conformally invariant integral equations: the method of moving spheres
}

Received August 14, 2003

\section{Introduction}

For $n \geq 3$, consider

$$
-\Delta u=n(n-2) u^{\frac{n+2}{n-2}} \quad \text { on } \mathbb{R}^{n} .
$$

It was proved by Gidas, Ni and Nirenberg [21] that any positive $C^{2}$ solution of (1) satisfying

$$
\liminf _{|x| \rightarrow \infty}|x|^{n-2} u(x)<\infty
$$

must be of the form

$$
u(x) \equiv\left(\frac{a}{1+a^{2}|x-\bar{x}|^{2}}\right)^{(n-2) / 2},
$$

where $a>0$ is some constant and $\bar{x} \in \mathbb{R}^{n}$.

Hypothesis (2) was removed by Caffarelli, Gidas and Spruck in [8]; this is important for applications. Such Liouville type theorems have been extended to general conformally invariant fully nonlinear equations by $\mathrm{Li}$ and $\mathrm{Li}([24]-[27])$; see also related works of Viaclovsky ([40]-[41]) and Chang, Gursky and Yang ([13]-[14]). The method used in [21], as well as in much of the above cited work, is the method of moving planes. The method of moving planes has become a very powerful tool in the study of nonlinear elliptic equations; see Aleksandrov [1], Serrin [38], Gidas, Ni and Nirenberg [21]-[22], Berestycki and Nirenberg [2], and others.

In [30], Li and Zhu gave a proof of the above mentioned theorem of Caffarelli, Gidas and Spruck using the method of moving spheres (i.e. the method of moving planes together with the conformal invariance), which fully exploits the conformal invariance of the problem and, as a result, captures the solutions directly rather than going through the usual procedure of proving radial symmetry of solutions and then classifying radial solutions. Significant simplifications to the proof in [30] have been made in Li and Zhang [29]. The method of moving spheres has been used in [24]-[27].

Liouville type theorems for various conformally invariant equations have received much attention; see, in addition to the above cited papers, [23], [17], [15], [33], [42] and [43].

Y. Y. Li: Department of Mathematics, Rutgers University, 110 Frelinghuysen Rd., Piscataway, NJ 08854, USA; e-mail: yyli@math.rutgers.edu 
In this paper we study some conformally invariant integral equations. Lieb proved in [31], among other things, that there exist maximizing functions, $f$, for the HardyLittlewood-Sobolev inequality on $\mathbb{R}^{n}$ :

$$
\left\|\int_{\mathbb{R}^{n}} \frac{f(y)}{|\cdot-y|^{\lambda}} d y\right\|_{L^{q}\left(\mathbb{R}^{n}\right)} \leq N_{p, \lambda, n}\|f\|_{L^{p}\left(\mathbb{R}^{n}\right)},
$$

with $N_{p, \lambda, n}$ being the sharp constant and $1 / p+\lambda / n=1+1 / q, 1<p, q, n / \lambda<\infty$, $n \geq 1$. When $p=q^{\prime}=q /(q-1)$ or $p=2$ or $q=2, N_{p, \lambda, n}$ and the maximizing $f$ 's are explicitly evaluated. When $p=q^{\prime}$, i.e., $p=2 n /(2 n-\lambda)$ and $q=2 n / \lambda$, the Euler-Lagrange equation for a maximizing $f$ is, modulo a positive constant multiple,

$$
f(x)^{p-1}=\int_{\mathbb{R}^{n}} \frac{f(y)}{|x-y|^{\lambda}} d y .
$$

Writing $\lambda=n-\alpha$ and $u=f^{p-1}$, we have $0<\alpha<n$, and equation 3 becomes

$$
u(x)=\int_{\mathbb{R}^{n}} \frac{u(y)^{\frac{n+\alpha}{n-\alpha}}}{|x-y|^{n-\alpha}} d y, \quad \forall x \in \mathbb{R}^{n} .
$$

As mentioned above, maximizing solutions $f$ of $[3]$ are classified in [31] and they are, in terms of $u$, of the form

$$
u(x) \equiv\left(\frac{a}{d+|x-\bar{x}|^{2}}\right)^{(n-\alpha) / 2},
$$

where $a, d>0$ and $\bar{x} \in \mathbb{R}^{n}$. Of course, $a$ is a fixed constant depending only on $n$ and $\alpha$, while $d$ and $\bar{x}$ are free.

Equation (4), or (3), is conformally invariant in the following sense. Let $v$ be a positive function on $\mathbb{R}^{n}$. For $x \in \mathbb{R}^{n}$ and $\lambda>0$, we define

$$
v_{x, \lambda}(\xi)=\left(\frac{\lambda}{|\xi-x|}\right)^{n-\alpha} v\left(\xi^{x, \lambda}\right), \quad \xi \in \mathbb{R}^{n},
$$

where

$$
\xi^{x, \lambda}=x+\frac{\lambda^{2}(\xi-x)}{|\xi-x|^{2}} .
$$

Then, if $u$ is a solution of (4), so is $u_{x, \lambda}$ for any $x \in \mathbb{R}^{n}$ and $\lambda>0$. The conformal invariance of (4) was used in [31]. More studies on issues concerning the Hardy-LittlewoodSobolev inequality, among other things, were made by Carlen and Loss in [9]-[12], where the conformal invariance of the problem was further exploited.

After classifying all maximizing solutions of (3), Lieb raised the beautiful question (page 361 of [31]) on the (essential) uniqueness of solutions of (3), or equivalently, of (4). He produced (page 363 of [31]) a nontrivial $2 n$-parameter family of solutions of equation (3), or (4), which are not as regular as the maximizers. For instance, modulo a positive constant, $|x|^{(\alpha-n) / 2}$ is a solution of $(4)$.

In a recent paper, Chen, $\mathrm{Li}$ and $\mathrm{Ou}$ established the following result which answers the question of Lieb in the class of $L_{\mathrm{loc}}^{\infty}\left(\mathbb{R}^{n}\right)$. 
Theorem 1.1 ([18]). Let $u \in L_{\mathrm{loc}}^{\infty}\left(\mathbb{R}^{n}\right)$ be a positive function satisfying 4 . Then $u$ is given by (5) for some constants $a, d>0$ and some $\bar{x} \in \mathbb{R}^{n}$.

In an earlier version of the present paper ([28, version one]), we gave a simpler proof of Theorem 1.1] The proof, in the spirit of [30] and [29] and following Section 2 of [29], fully exploits the conformal invariance of the integral equation. It is different from the one in [18]. In particular, we do not follow the usual procedure of proving radial symmetry of solutions and then classifying radial solutions, and we do not need to distinguish $n \geq 2$ and $n=1$. For the method of moving spheres or moving planes, there are, roughly speaking, three steps: one is to get started with the procedure, second is to prove that the function and the reflected one coincide if the procedure stops, and the third is to handle the case when the procedure never stops. Our arguments are also different for handling these steps. This proof is presented in Section 2.

Lieb pointed out to us that his question also concerns functions which are not in $L_{\text {loc }}^{\infty}\left(\mathbb{R}^{n}\right)$. In particular, it is not known a priori that maximizers are in $L_{\text {loc }}^{\infty}\left(\mathbb{R}^{n}\right)$. This has led us to study the question further and to establish

Theorem 1.2. For $n \geq 1$ and $0<\alpha<n$, let $u \in L_{\text {loc }}^{2 n /(n-\alpha)}\left(\mathbb{R}^{n}\right)$ be a positive solution of (4). Then $u \in C^{\infty}\left(\mathbb{R}^{n}\right)$.

An answer to the question of Lieb is therefore known in the class $L_{\text {loc }}^{2 n /(n-\alpha)}\left(\mathbb{R}^{n}\right)$. The above mentioned solution $|x|^{(\alpha-n) / 2}$ does not belong to $L_{\text {loc }}^{2 n /(n-\alpha)}\left(\mathbb{R}^{n}\right)$, though it belongs to $L_{\mathrm{loc}}^{t}\left(\mathbb{R}^{n}\right)$ for any $t<2 n /(n-\alpha)$. The question remains unanswered for the class $L_{\text {loc }}^{t}\left(\mathbb{R}^{n}\right)$ for $t<2 n /(n-\alpha)$. See [34] and the references therein for related results.

In the process of proving Theorem 1.2 , we have established the following result which should be of independent interest.

For $n \geq 1$ and $0<\alpha<n$, let $V \in L^{n / \alpha}\left(B_{3}\right)$ be a nonnegative function, and set

$$
\delta(V):=\|V\|_{L^{n / \alpha}\left(B_{3}\right)} \text {. }
$$

Theorem 1.3. For $n \geq 1,0<\alpha<n$ and $v>r>n /(n-\alpha)$, there exist positive constants $\bar{\delta}<1$ and $C \geq 1$, depending only on $n, \alpha, r$ and $v$, such that for any $0 \leq V \in$ $L^{n / \alpha}\left(B_{3}\right)$ with $\delta(V) \leq \bar{\delta}, h \in L^{v}\left(B_{2}\right)$, and $0 \leq u \in L^{r}\left(B_{3}\right)$ satisfying

$$
u(x) \leq \int_{B_{3}} \frac{V(y) u(y)}{|x-y|^{n-\alpha}} d y+h(x), \quad x \in B_{2},
$$

we have

$$
\|u\|_{L^{v}\left(B_{1 / 2}\right)} \leq C\left(\|u\|_{L^{r}\left(B_{3}\right)}+\|h\|_{L^{v}\left(B_{2}\right)}\right) \text {. }
$$

Corollary 1.1. For $n \geq 1,0<\alpha<n, v>r>n /(n-\alpha)$ and $R_{2}>R_{1}>0$, let $0 \leq V \in L^{n / \alpha}\left(B_{R_{2}}\right), h \in L^{\nu}\left(B_{R_{1}}\right)$ and $0 \leq u \in L^{r}\left(B_{R_{2}}\right)$ satisfy

$$
u(x) \leq \int_{B_{R_{2}}} \frac{V(y) u(y)}{|x-y|^{n-\alpha}} d y+h(x), \quad x \in B_{R_{1}} .
$$

Then, for some $\epsilon>0, u \in L^{v}\left(B_{\epsilon}\right)$.

Remark 1.1. After we proved Theorems 1.2 and 1.3 in [28, version two], a revision of [18] was made which included another proof of Theorem 1.2. 
For $\alpha=2$ and $n \geq 3$, Theorem 1.3 is essentially equivalent to a result of Brezis and Kato (Theorem 2.3 in [6]), so it can be viewed as an integral equation analogue of their theorem. When informed of Theorem 1.3. Brezis kindly pointed out that it is similar to, though not the same as, Lemma A.1 in [7]. Indeed, our proof of the theorem makes use of special properties of the potential $|x|^{\alpha-n}$, and it is not clear to us at this point whether the conclusion of the theorem still holds if we replace $|x|^{\alpha-n}$ by any $Y \in L_{w}^{n /(n-\alpha)}$, the weak $L^{n /(n-\alpha)}$ space, as in Lemma A.1 of [7]. Theorem 1.2. Theorem 1.3 and Corollary 1.1 are established in Section 2.

We also study some equations similar to (4), though they do not have the same kind of conformal invariance property. For $n \geq 1,0<\alpha<n$ and $\mu>0$, let $u$ be a positive Lebesgue measurable function in $\mathbb{R}^{n}$ satisfying

$$
u(x)=\int_{\mathbb{R}^{n}} \frac{u(y)^{\mu}}{|x-y|^{n-\alpha}} d y, \quad \forall x \in \mathbb{R}^{n} .
$$

Theorem 1.4. Let $n \geq 1$ and $0<\alpha<n$.

(i) For $0<\mu<n /(n-\alpha)$, equation (11) does not have any positive Lebesgue measurable solution $u$, unless $u \equiv \infty$.

(ii) For $n /(n-\alpha) \leq \mu<(n+\alpha) /(n-\alpha)$, equation (11) does not have any positive solution $u \in L_{\text {loc }}^{n(\mu-1) / \alpha}\left(\mathbb{R}^{n}\right)$.

For $\mu>(n+\alpha) /(n-\alpha)$, we know from Lemma 4.2 that if $u$ is a positive solution in $L_{\text {loc }}^{n(\mu-1) / \alpha}\left(\mathbb{R}^{n}\right)$, then $u$ must be in $C^{\infty}\left(\mathbb{R}^{n}\right)$. Theorem 1.4 is proved in Section 4.

In [24]-[27], all conformally invariant second order fully nonlinear equations are classified and Liouville type theorems are established for the elliptic ones. It would be interesting to identify as many as possible conformally invariant integral equations for which (essential) uniqueness of solutions can be obtained. One class of such equations, similar to 4 , is

$$
u(x)=\int_{\mathbb{R}^{n}}|x-y|^{p} u(y)^{-(2 n+p) / p} d y, \quad \forall x \in \mathbb{R}^{n},
$$

where $n \geq 1$ and $p>0$. We study more general equations, similar to 111 , including those which are not conformally invariant.

For $n \geq 1$ and $p, q>0$, let $u$ be a nonnegative Lebesgue measurable function in $\mathbb{R}^{n}$ satisfying

$$
u(x)=\int_{\mathbb{R}^{n}}|x-y|^{p} u(y)^{-q} d y, \quad \forall x \in \mathbb{R}^{n} .
$$

Theorem 1.5. For $n \geq 1, p>0$ and $0<q \leq 1+2 n / p$, let $u$ be a nonnegative Lebesgue measurable function in $\mathbb{R}^{n}$ satisfying (12). Then $q=1+2 n / p$ and, for some constants $a, d>0$ and some $\bar{x} \in \mathbb{R}^{n}$,

$$
u(x) \equiv\left(\frac{d+|x-\bar{x}|^{2}}{a}\right)^{p / 2}
$$

Remark 1.2. For some $a=a(n, p)>0$, 13 indeed solves 12 with $q=1+2 n / p$. This is proved in Appendix A. The argument also shows that, modulo a constant, (5) is a 
solution of (4), a known fact whose proofs can be found in [39, page 131], [31], and, for $n \geq 2$, in [36]. Our proof is different.

The proof of Theorem 1.5, similar to our proof of Theorem 1.1. is given in Section 5. It turns out that for $n=3, p=1$ and $q=7$, integral equation (12) is associated with some fourth order conformal covariant operator on 3-dimensional compact Riemannian manifolds, arising from the study of conformal geometry. See, e.g., Paneitz [37], Fefferman and Graham [19], Branson [3] and Chang and Yang [16].

Question 1. Is equation (12), in the case $p>0$ and $q=1+2 n / p$, associated with some kind of pseudo-differential conformal covariant operators on n-dimensional compact Riemannian manifolds, the same way the case $n=3, p=1$ and $q=7$ is associated with the above mentioned fourth order conformal covariant operator?

After posting [28, version one] on the Archive and essentially completing the proof of Theorem 1.5. we became aware of some recent work of $\mathrm{Xu}$ [44] where he proved Theorem 1.5 in the special case $n=3, p=1$ and $u \in C^{4}\left(\mathbb{R}^{3}\right)$. He also proved in the same paper that for $n=3, p=1$ and $q>7(=1+2 n / p)$, equation (12) does not admit any nonnegative solution $u$ in $C^{4}\left(\mathbb{R}^{3}\right)$. Radial solutions of the biharmonic equations corresponding to (12) with $n=3$ and $p=1$ were studied by McKenna and Reichel in [35].

Question 2. Is it true that for all $n \geq 1, p>0$ and $q>1+2 n / p$ equation (12) does not admit any positive solutions?

We point out that if we consider the integral equations of the form

$$
u(x)=\int_{\mathbb{R}^{n}} G(|x-y|, u(y)) d y, \quad u>0, \quad \forall x \in \mathbb{R}^{n},
$$

and consider the transformation of the form

$$
u_{x, \lambda}(\xi)=h\left(\left(\frac{\lambda}{|\xi-x|}\right)^{2 n}\right) u\left(\xi^{x, \lambda}\right),
$$

where $\xi^{x, \lambda}$ is given by 7 , and wish that

$$
h\left(\left(\frac{\lambda}{|\xi-x|}\right)^{2 n}\right) \int_{\mathbb{R}^{n}} G\left(\left|\xi^{x, \lambda}-y\right|, u(y)\right) d y \equiv \int_{\mathbb{R}^{n}} G\left(|\xi-z|, u_{x, \lambda}(z)\right) d z
$$

for all $x, \xi \in \mathbb{R}^{n}, \lambda>0$ and all positive functions $u$, then we are only led to equation (44) and equation (12) with $q=1+2 n / p$ together with the transformations we use in the paper. Note that condition (15) guarantees that whenever $u$ is a solution of (14) so is $u_{x, \lambda}$ for all $x \in \mathbb{R}^{n}$ and $\lambda>0$. The quantity $(\lambda /|\xi-x|)^{2 n}$ is the Jacobian of the conformal transformation $\xi \mapsto \xi^{x, \lambda}$.

It looks worthwhile to study equation (12) on a bounded domain (existence of solutions, etc.). In this connection, we draw the reader's attention to some works of Brezis and Cabre [4] and Brezis, Dupaigne and Tesei [5]. 


\section{Proof of Theorem 1.3, Corollary 1.1 and Theorem 1.2}

In this section we prove Theorem 1.3 . Let

$$
\xi(x):=\int_{B_{3}} \frac{V(y) u(y)}{|x-y|^{n-\alpha}} d y+h(x)-u(x) \geq 0, \quad x \in B_{2} .
$$

Then

$$
u(x)=(L u)(x)+f(x)+h(x)-\xi(x), \quad x \in B_{2},
$$

where

$$
(L u)(x)=\int_{B_{2}} \frac{V(y) u(y)}{|x-y|^{n-\alpha}} d y, \quad x \in B_{2},
$$

and

$$
f(x)=\int_{2<|y|<3} \frac{V(y) u(y)}{|x-y|^{n-\alpha}} d y .
$$

Let $p$ be determined by $1 / r=1 / p-\alpha / n$. Then $p>1$ and therefore, by the property of the Riesz potential (see, e.g., Theorem 1 on page 119 of [39]),

$$
\begin{aligned}
\|L u\|_{L^{r}\left(B_{2}\right)} & \leq C\|V u\|_{L^{p}\left(B_{2}\right)}=C\left(\left\|V^{p} u^{p}\right\|_{L^{1}\left(B_{2}\right)}\right)^{1 / p} \\
& \leq C\left(\left\|V^{p}\right\|_{L^{r /(r-p)\left(B_{2}\right)}}\left\|u^{p}\right\|_{L^{r / p}\left(B_{2}\right)}\right)^{1 / p} \leq C\|V\|_{L^{n / \alpha}\left(B_{2}\right)}\|u\|_{L^{r}\left(B_{2}\right)},
\end{aligned}
$$

where $C$ depends on $\alpha, n$ and $r$. Similarly

$$
\|f\|_{L^{r}\left(B_{2}\right)} \leq C\|V\|_{L^{n / \alpha}\left(B_{3}\right)}\|u\|_{L^{r}\left(B_{3}\right)} .
$$

It follows, using also the fact $u, \xi \geq 0$, that

$$
\|\xi\|_{L^{r}\left(B_{2}\right)} \leq C\|V\|_{L^{n / \alpha\left(B_{3}\right)}}\|u\|_{L^{r}\left(B_{3}\right)}+C\|h\|_{L^{r}\left(B_{2}\right)} .
$$

For $i=1,2, \ldots$, let

$$
\begin{gathered}
G_{i}(z)=\min \left(\frac{1}{|z|^{n-\alpha}}, i\right), \quad u_{i}(z)=\min (u(z), i), \\
\xi_{i}(x)=\min (\xi(x), i), \quad f_{i}(x)=\int_{2<|y|<3} G_{i}(x-y) V(y) u(y) d y .
\end{gathered}
$$

We now give some preliminary estimates on $\left\{f_{i}\right\}$ :

Lemma 2.1. There exists some constant $C$, depending only on $n$ and $\alpha$, such that

$$
\left\|f_{i}\right\|_{L^{\infty}\left(B_{1}\right)} \leq C\|u\|_{L^{r}\left(B_{3}\right)}, \quad\left\|f_{i}\right\|_{L^{r}\left(B_{2}\right)} \leq C\|u\|_{L^{r}\left(B_{3}\right)} .
$$

Moreover, for any $p<r$,

$$
\lim _{i \rightarrow \infty}\left\|f_{i}-f\right\|_{L^{p}\left(B_{2}\right)}=0 .
$$


Proof. The first inequality in (20) follows easily:

$$
\left\|f_{i}\right\|_{L^{\infty}\left(B_{1}\right)} \leq\|f\|_{L^{\infty}\left(B_{1}\right)} \leq C(n, \alpha) \int_{2<|y|<3} V(y) u(y) d y \leq C(n, \alpha)\|u\|_{L^{r}\left(B_{3}\right)} .
$$

Note that we have used the hypothesis $\|V\|_{L^{n / \alpha}\left(B_{3}\right)} \leq \bar{\delta}<1$. The second inequality in 20) follows from (18).

By the Fubini theorem,

$$
\lim _{i \rightarrow \infty}\left\|f_{i}-f\right\|_{L^{1}\left(B_{2}\right)} \leq \lim _{i \rightarrow \infty}\left\|G_{i}(\cdot)-\frac{1}{|\cdot|^{n-\alpha}}\right\|_{L^{1}\left(B_{5}\right)} \int_{2<|y|<3} V(y) u(y) d y=0 .
$$

We deduce 21] from this and the second inequality in 20 using Hölder's inequality.

Consider the following integral equation on $w$ :

$$
w(x)=\left(L_{i} w\right)(x)+f_{i}(x)+h(x)-\xi_{i}(x), \quad x \in B_{2},
$$

where

$$
\left(L_{i} w\right)(x):=\int_{|y|<2} G_{i}(x-y) V(y) w(y) d y .
$$

Lemma 2.2. For $r \leq q \leq v$, there exist some $0<\bar{\delta}<1$ and $C \geq 1$, depending only on $\alpha, n, r$ and $q$, such that if $0<\delta(V) \leq \bar{\delta}$, then, for all $i$, there exists $w_{i} \in L^{q}\left(B_{2}\right)$ solving 22. with $w=w_{i}$, satisfying

$$
\left\|w_{i}\right\|_{L^{r}\left(B_{2}\right)}+\left\|w_{i}^{+}\right\|_{L^{q}\left(B_{1 / 2}\right)} \leq C\left(\|u\|_{L^{r}\left(B_{3}\right)}+\|h\|_{L^{r}\left(B_{2}\right)}\right),
$$

where $w_{i}^{+}(x)=\max \left(w_{i}(x), 0\right)$.

Proof. Define, for $w \in L^{q}\left(B_{2}\right)$,

$$
\left(T_{i} w\right)(x)=\left(L_{i} w\right)(x)+f_{i}(x)+h(x)-\xi_{i}(x), \quad x \in B_{2} .
$$

Clearly, $L_{i}, T_{i}: L^{q}\left(B_{2}\right) \rightarrow L^{q}\left(B_{2}\right)$.

Let $p$ be determined by $1 / q=1 / p-\alpha / n$. Then, using the property of the Riesz potential as in (17), we obtain

$$
\left\|L_{i} w\right\|_{L^{q}\left(B_{2}\right)} \leq\|L(|w|)\|_{L^{q}\left(B_{2}\right)} \leq C\|V\|_{L^{n / \alpha}\left(B_{2}\right)}\|w\|_{L^{q}\left(B_{2}\right)} \leq C \bar{\delta}\|w\|_{L^{q}\left(B_{2}\right)} .
$$

Here and below (various) constants $C \geq 1$ depend only on $r, q, \alpha$ and $n$. Thus

$$
\left\|T_{i} w\right\|_{L^{q}\left(B_{2}\right)} \leq C \bar{\delta}\|w\|_{L^{q}\left(B_{2}\right)}+\left\|f_{i}\right\|_{L^{q}\left(B_{2}\right)}+\|h\|_{L^{q}\left(B_{2}\right)}+\left\|\xi_{i}\right\|_{L^{q}\left(B_{2}\right)},
$$

and

$$
\left\|T_{i}(w-v)\right\|_{L^{q}\left(B_{2}\right)} \leq\left\|L_{i}(w-v)\right\|_{L^{q}\left(B_{2}\right)} \leq C \bar{\delta}\|w-v\|_{L^{q}\left(B_{2}\right)} .
$$

Fix some positive $\bar{\delta}$ with $C \bar{\delta} \leq 1 / 2$ and set

$$
E_{i}=\left\{w \in L^{q}\left(B_{2}\right) \mid\|w\|_{L^{q}\left(B_{2}\right)} \leq 2\left(\left\|f_{i}\right\|_{L^{q}\left(B_{2}\right)}+\|h\|_{L^{q}\left(B_{2}\right)}+\left\|\xi_{i}\right\|_{L^{q}\left(B_{2}\right)}\right)\right\} \subset L^{q}\left(B_{2}\right) .
$$


Then $T_{i}$ maps $E_{i}$ to itself and is a contraction map. So there exists some $w_{i} \in E_{i}$ such that $T_{i}\left(w_{i}\right)=w_{i}$, i.e.,

$$
w_{i}(x)=\int_{|y|<2} G_{i}(x-y) V(y) w_{i}(y) d y+f_{i}(x)+h(x)-\xi_{i}(x), \quad x \in B_{2} .
$$

Taking $q=r$ in (24), we deduce from 25 and 20 that

$$
\left\|w_{i}\right\|_{L^{r}\left(B_{2}\right)} \leq \frac{1}{2}\left\|w_{i}\right\|_{L^{r}\left(B_{2}\right)}+\left\|f_{i}\right\|_{L^{r}\left(B_{2}\right)}+\|h\|_{L^{r}\left(B_{2}\right)}+\|\xi\|_{L^{r}\left(B_{2}\right)} .
$$

The estimate of $\left\|w_{i}\right\|_{L^{r}\left(B_{2}\right)}$ in (23) follows from this, in view of (19) and the second inequality in 20 .

Next we establish the second inequality in 23]. For $0<t<s<1$, we have, by (25),

$$
w_{i}^{+}(x) \leq I_{i}(x)+I I_{i}(x)+f_{i}(x)+h(x),
$$

where

$$
I_{i}(x)=\int_{|y|<s} \frac{V(y) w_{i}^{+}(y)}{|x-y|^{n-\alpha}} d y, \quad I I_{i}(x)=\int_{s<|y|<2} \frac{V(y) w_{i}^{+}(y)}{|x-y|^{n-\alpha}} d y .
$$

By the property of the Riesz potential,

$$
\begin{aligned}
\left\|I_{i}\right\|_{L^{q}\left(B_{t}\right)} & \leq C\left\|V w_{i}^{+}\right\|_{L^{p}\left(B_{s}\right)} \leq C\|V\|_{L^{n / \alpha}\left(B_{s}\right)}\left\|w_{i}^{+}\right\|_{L^{q}\left(B_{s}\right)} \\
& \leq C \bar{\delta}\left\|w_{i}^{+}\right\|_{L^{q}\left(B_{s}\right)} \leq \frac{1}{2}\left\|w_{i}^{+}\right\|_{L^{q}\left(B_{s}\right)} .
\end{aligned}
$$

Using the estimate of $\left\|w_{i}\right\|_{L^{r}\left(B_{2}\right)}$ in 23 yields

$$
\begin{aligned}
\left\|I I_{i}\right\|_{L^{q}\left(B_{t}\right)} & \leq C(s-t)^{\alpha-n} \int_{s<|y|<2} V(y) w_{i}^{+}(y) d y \\
& \leq C(s-t)^{\alpha-n}\left\|w_{i}\right\|_{L^{r}\left(B_{2}\right)} \leq C(s-t)^{\alpha-n}\left(\|u\|_{L^{r}\left(B_{3}\right)}+\|h\|_{L^{r}\left(B_{2}\right)}\right) .
\end{aligned}
$$

With 20) and the above estimates, we have, for all $0<t<s<1$,

$$
\left\|w_{i}^{+}\right\|_{L^{q}\left(B_{t}\right)} \leq \frac{1}{2}\left\|w_{i}^{+}\right\|_{L^{q}\left(B_{s}\right)}+C(s-t)^{\alpha-n}\left(\|u\|_{L^{r}\left(B_{3}\right)}+\|h\|_{L^{r}\left(B_{2}\right)}\right) .
$$

By a calculus lemma (see, e.g., page 32 of [20]), we have, for a possibly larger $C$, still depending only on $r, q, \alpha$ and $n$,

$$
\left\|w_{i}^{+}\right\|_{L^{q}\left(B_{t}\right)} \leq C(s-t)^{\alpha-n}\left(\|u\|_{L^{r}\left(B_{3}\right)}+\|h\|_{L^{r}\left(B_{2}\right)}\right), \quad \forall 0<t<s<1 .
$$

The estimate of $\left\|w_{i}^{+}\right\|_{L^{q}\left(B_{1 / 2}\right)}$ in 23 follows from the above. Lemma 2.2 is proved.

Proof of Theorem 1.3. For any $r<q \leq v$, let $\bar{\delta}>0$ and $\left\{w_{i}\right\} \in L^{q}\left(B_{2}\right)$ be given by Lemma 2.2. Since

$$
\int_{|y|<2} V(y) w_{i}(y) d y \leq C\|V\|_{L^{n / \alpha}\left(B_{2}\right)}\left\|w_{i}\right\|_{L^{r}\left(B_{2}\right)} \leq C
$$

for some $C$ independent of $i$, we have

$$
\lim _{|z| \rightarrow 0} \sup _{i}\left\|\left(L_{i} w_{i}\right)(\cdot+z)-\left(L_{i} w_{i}\right)(\cdot)\right\|_{L^{1}\left(B_{2}\right)}=0 .
$$

Therefore $\left\{L_{i} w_{i}\right\}$ is precompact in $L^{1}\left(B_{2}\right)$. 
We know from Lemma 2.1 that $\left\{f_{i}\right\}$ converges to $f$ in $L^{1}\left(B_{2}\right)$. So $\left\{w_{i}\right\}$ is precompact in $L^{1}\left(B_{2}\right)$. After passing to a subsequence, $w_{i} \rightarrow w$ in $L^{1}\left(B_{2}\right)$. In view of 23, $w \in$ $L^{r}\left(B_{2}\right), w_{i} \rightarrow w$ in $L^{p}\left(B_{2}\right)$ for all $p<r, w^{+} \in L^{q}\left(B_{1 / 2}\right)$, and

$$
\left\|w^{+}\right\|_{L^{q}\left(B_{1 / 2}\right)} \leq C\left(\|u\|_{L^{r}\left(B_{3}\right)}+\|h\|_{L^{v}\left(B_{2}\right)}\right) .
$$

It follows that $L_{i} w_{i} \rightarrow L w$ in $L^{1}\left(B_{2}\right)$. Thus,

$$
w(x)=\int_{|y|<2} \frac{V(y) w(y)}{|x-y|^{n-\alpha}} d y+f(x)+h(x)-\xi(x), \quad \text { a.e. } x \in B_{2} .
$$

Taking the difference of this and $(16)$, we obtain

$$
(u-w)(x)=\int_{|y|<2} \frac{V(y)(u-w)(y)}{|x-y|^{n-\alpha}} d y, \quad \text { a.e. } x \in B_{2} .
$$

By the usual estimates and using $0<\delta(V) \leq \bar{\delta}$ and $C \bar{\delta} \leq 1 / 2$, we infer that

$$
\|u-w\|_{L^{r}\left(B_{2}\right)} \leq C \bar{\delta}\|u-w\|_{L^{r}\left(B_{2}\right)} \leq \frac{1}{2}\|u-w\|_{L^{r}\left(B_{2}\right)} .
$$

It follows that $u=w$ a.e. in $B_{2}$. Theorem 1.3 follows from 26.

Proof of Corollary [.1. For $\epsilon>0$ small, let

$$
u_{\epsilon}(x)=\epsilon^{(n-\alpha) / 2} u(\epsilon x), \quad V_{\epsilon}(x)=\epsilon^{\alpha} V(\epsilon x), \quad x \in B_{3},
$$

and

$$
h_{\epsilon}(x)=\epsilon^{(n-\alpha) / 2} \int_{3 \epsilon<|y|<R_{2}} \frac{V(y) u(y)}{|\epsilon x-y|^{n-\alpha}} d y+\epsilon^{(n-\alpha) / 2} h(\epsilon x) .
$$

Then

$$
u_{\epsilon}(x) \leq \int_{B_{3}} \frac{V_{\epsilon}(y) u_{\epsilon}(y)}{|x-y|^{n-\alpha}} d y+h_{\epsilon}(x), \quad x \in B_{2} .
$$

Clearly, $u_{\epsilon} \in L^{r}\left(B_{3}\right)$ and $h_{\epsilon} \in L^{\nu}\left(B_{2}\right)$. Let $\bar{\delta}>0$ be the number in Theorem 1.3, and fix some small $\epsilon>0$ so that

$$
\left\|V_{\epsilon}\right\|_{L^{n / \alpha}\left(B_{3}\right)}=\|V\|_{L^{n / \alpha}\left(B_{3 \epsilon}\right)}<\bar{\delta} .
$$

Applying Theorem 1.3 to $u_{\epsilon}$, we have $u_{\epsilon} \in L^{\nu}\left(B_{1 / 2}\right)$, i.e. $u \in L^{\nu}\left(B_{\epsilon / 2}\right)$.

Proof of Theorem 1.2 Since $u \in L_{\text {loc }}^{2 n /(n-\alpha)}\left(\mathbb{R}^{n}\right)$, we have, by 4 , for some $|\bar{x}|<1$,

$$
\int_{|y|>2} \frac{u(y)^{\frac{n+\alpha}{n-\alpha}}}{|y|^{n-\alpha}} d y \leq C \int_{|y|>2} \frac{u(y)^{\frac{n+\alpha}{n-\alpha}}}{|\bar{x}-y|^{n-\alpha}} d y \leq \int_{\mathbb{R}^{n}} \frac{u(y)^{\frac{n+\alpha}{n-\alpha}}}{|\bar{x}-y|^{n-\alpha}} d y=u(\bar{x})<\infty .
$$

For any $R>0$, we write

$$
u(x)=I_{R}(x)+I I_{R}(x):=\left(\int_{|y| \leq 2 R}+\int_{|y|>2 R}\right) \frac{u(y)^{\frac{n+\alpha}{n-\alpha}}}{|x-y|^{n-\alpha}} d y .
$$


Take

$$
V(x)=u(x)^{\frac{2 \alpha}{n-\alpha}}, \quad h(x)=\int_{|y|>2 R} \frac{u(y)^{\frac{n+\alpha}{n-\alpha}}}{|x-y|^{n-\alpha}} d y .
$$

Since $u \in L_{\text {loc }}^{2 n / n-\alpha}\left(\mathbb{R}^{n}\right)$, we have $V \in L_{\text {loc }}^{n / \alpha}\left(\mathbb{R}^{n}\right)$. By $27, h \in L^{\infty}\left(B_{R}\right)$. For any $v>$ $n /(n-\alpha)$, we have, by Corollary 1.1, $u \in L^{\nu}\left(B_{\epsilon(v)}\right)$ for some $\epsilon(v)>0$. Since any point can be taken as the origin, we have proved that $u \in L_{\text {loc }}^{v}\left(\mathbb{R}^{n}\right)$ for all $1<v<\infty$. By the Hölder inequality, $I_{R} \in L^{\infty}\left(B_{R}\right)$. By 27), we can differentiate $I I_{R}(x)$ under the integral sign for $|x|<R$, so $I I_{R} \in C^{\infty}\left(\mathbb{R}^{n}\right)$. Since $R$ is arbitrary, $u \in L_{\text {loc }}^{\infty}\left(\mathbb{R}^{n}\right)$. Back to (28), $I_{R}$ is at least Hölder continuous in $B_{R}$. Since $R>0$ is arbitrary, $u$ is Hölder continuous in $\mathbb{R}^{n}$. Now $u^{(n+\alpha) /(n-\alpha)}$ is Hölder continuous in $B_{2 R}$, the regularity of $I_{R}$ further improves and, by bootstrap, we eventually have $u \in C^{\infty}\left(\mathbb{R}^{n}\right)$.

\section{Proof of Theorem 1.1}

In this section we prove Theorem 1.1 As shown in the last paragraph of Section 2, $u \in$ $C^{\infty}\left(\mathbb{R}^{n}\right)$. By 4 and the Fatou lemma,

$$
\beta:=\liminf _{|x| \rightarrow \infty}|x|^{n-\alpha} u(x)=\liminf _{|x| \rightarrow \infty} \int_{\mathbb{R}^{n}} \frac{|x|^{n-\alpha} u(y)^{\frac{n+\alpha}{n-\alpha}}}{|x-y|^{n-\alpha}} d y \geq \int_{\mathbb{R}^{n}} u(y)^{\frac{n+\alpha}{n-\alpha}} d y>0 .
$$

For $x \in \mathbb{R}^{n}, \lambda>0$, and a positive function $v$ on $\mathbb{R}^{n}$, let $v_{x, \lambda}$ be given by (6). Making a change of variables

we have

$$
y=z^{x, \lambda}=x+\frac{\lambda^{2}(z-x)}{|z-x|^{2}}
$$

Thus

$$
d y=\left(\frac{\lambda}{|z-x|}\right)^{2 n} d z
$$

$$
\begin{aligned}
\int_{|y-x| \geq \lambda} \frac{v(y)^{\frac{n+\alpha}{n-\alpha}}}{\left|\xi^{x, \lambda}-y\right|^{n-\alpha}} d y & =\int_{|z-x| \leq \lambda} \frac{v\left(z^{x, \lambda}\right)^{\frac{n+\alpha}{n-\alpha}}}{\left|\xi^{x, \lambda}-z^{x, \lambda}\right|^{n-\alpha}}\left(\frac{\lambda}{|z-x|}\right)^{2 n} d z \\
& =\int_{|z-x| \leq \lambda} \frac{v_{x, \lambda}(z)^{\frac{n+\alpha}{n-\alpha}}}{\left|\xi^{x, \lambda}-z^{x, \lambda}\right|^{n-\alpha}}\left(\frac{\lambda}{|z-x|}\right)^{n-\alpha} d z
\end{aligned}
$$

Since

we have

$$
\frac{|z-x|}{\lambda} \frac{|\xi-x|}{\lambda}\left|\xi^{x, \lambda}-z^{x, \lambda}\right|=|\xi-z|
$$

$$
\left(\frac{\lambda}{|\xi-x|}\right)^{n-\alpha} \int_{|y-x| \geq \lambda} \frac{v(y)^{\frac{n+\alpha}{n-\alpha}}}{\left|\xi^{x, \lambda}-y\right|^{n-\alpha}} d y=\int_{|z-x| \leq \lambda} \frac{v_{x, \lambda}(z)^{\frac{n+\alpha}{n-\alpha}}}{|\xi-z|^{n-\alpha}} d z
$$

Similarly,

$$
\left(\frac{\lambda}{|\xi-x|}\right)^{n-\alpha} \int_{|y-x| \leq \lambda} \frac{v(y)^{\frac{n+\alpha}{n-\alpha}}}{\left|\xi^{x, \lambda}-y\right|^{n-\alpha}} d y=\int_{|z-x| \geq \lambda} \frac{v_{x, \lambda}(z)^{\frac{n+\alpha}{n-\alpha}}}{|\xi-z|^{n-\alpha}} d z .
$$


For a positive solution $u$ of (4), applying (31) and (32) with $v=u$ and $v=u_{x, \lambda}$, and using the fact that $\left(\xi^{x, \lambda}\right)^{x, \lambda}=\xi$ and $\left(v_{x, \lambda}\right)_{x, \lambda} \equiv v$, we obtain

$$
u_{x, \lambda}(\xi)=\int_{\mathbb{R}^{n}} \frac{u_{x, \lambda}(z)^{\frac{n+\alpha}{n-\alpha}}}{|\xi-z|^{n-\alpha}} d z, \quad \forall \xi \in \mathbb{R}^{n},
$$

and

$$
u(\xi)-u_{x, \lambda}(\xi)=\int_{|z-x| \geq \lambda} K(x, \lambda ; \xi, z)\left[u(z)^{\frac{n+\alpha}{n-\alpha}}-u_{x, \lambda}(z)^{\frac{n+\alpha}{n-\alpha}}\right] d z
$$

where

It is elementary to check that

$$
K(x, \lambda ; \xi, z)=\frac{1}{|\xi-z|^{n-\alpha}}-\left(\frac{\lambda}{|\xi-x|}\right)^{n-\alpha} \frac{1}{\left|\xi^{x, \lambda}-z\right|^{n-\alpha}} .
$$

$$
K(x, \lambda ; \xi, z)>0, \quad \forall|\xi-x|,|z-x|>\lambda>0 .
$$

Formula (33) is the conformal invariance of the integral equation (4) (see [31] and [32]).

Lemma 3.1. For $x \in \mathbb{R}^{n}$, there exists $\lambda_{0}(x)>0$ such that

$$
u_{x, \lambda}(y) \leq u(y), \quad \forall 0<\lambda<\lambda_{0}(x),|y-x| \geq \lambda .
$$

Proof. The proof is essentially the same as that of Lemma 2.1 in [29]. For the reader's convenience, we include the details. Without loss of generality we may assume $x=0$, and we use the notation $u_{\lambda}=u_{0, \lambda}$.

Since $\alpha<n$ and $u$ is a positive $C^{1}$ function, there exists $r_{0}>0$ such that

$$
\nabla_{y}\left(|y|^{(n-\alpha) / 2} u(y)\right) \cdot y>0, \quad \forall 0<|y|<r_{0} .
$$

Consequently,

$$
u_{\lambda}(y)<u(y), \quad \forall 0<\lambda<|y|<r_{0} .
$$

By 29] and the positivity and continuity of $u$,

$$
u(z) \geq \frac{1}{C\left(r_{0}\right)|z|^{n-\alpha}} \quad \forall|z| \geq r_{0} .
$$

For small $\lambda_{0} \in\left(0, r_{0}\right)$ and for $0<\lambda<\lambda_{0}$,

$$
u_{\lambda}(y)=\left(\frac{\lambda}{|y|}\right)^{n-\alpha} u\left(\frac{\lambda^{2} y}{|y|^{2}}\right) \leq\left(\frac{\lambda_{0}}{|y|}\right)^{n-\alpha} \sup _{B_{r_{0}}} u \leq u(y), \quad \forall|y| \geq r_{0} .
$$

Estimate 35, with $x=0$ and $\lambda_{0}(x)=\lambda_{0}$, follows from 36 and the above.

Define, for $x \in \mathbb{R}^{n}$,

$$
\bar{\lambda}(x)=\sup \left\{\mu>0\left|u_{x, \lambda}(y) \leq u(y) \forall 0<\lambda<\mu,\right| y-x \mid \geq \lambda\right\} .
$$

Lemma 3.2. If $\bar{\lambda}(\bar{x})<\infty$ for some $\bar{x} \in \mathbb{R}^{n}$, then

$$
u_{\bar{x}, \bar{\lambda}(\bar{x})} \equiv u \quad \text { on } \mathbb{R}^{n} .
$$


Proof. Without loss of generality, we may assume $\bar{x}=0$, and we use notations $\bar{\lambda}=$ $\bar{\lambda}(0), u_{\lambda}=u_{0, \lambda}$. By the definition of $\bar{\lambda}$,

$$
u_{\bar{\lambda}}(y) \leq u(y), \quad \forall|y| \geq \bar{\lambda} .
$$

By 34 , with $x=0$ and $\lambda=\bar{\lambda}$, and the positivity of the kernel, either $u_{\bar{\lambda}}(y)=u(y)$ for all $|y| \geq \bar{\lambda}$-then we are done-or $u_{\bar{\lambda}}(y)<u(y)$ for all $|y|>\bar{\lambda}$, which we assume below. By the Fatou lemma,

$$
\begin{aligned}
\liminf _{|y| \rightarrow \infty}|y|^{n-\alpha}\left(u-u_{\bar{\lambda}}\right)(y) & =\liminf _{|y| \rightarrow \infty} \int_{|z| \geq \bar{\lambda}}|y|^{n-\alpha} K(0, \bar{\lambda} ; y, z)\left[u(z)^{\frac{n+\alpha}{n-\alpha}}-u_{\bar{\lambda}}(z)^{\frac{n+\alpha}{n-\alpha}}\right] d z \\
& \geq \int_{|z| \geq \bar{\lambda}}\left(1-\left(\frac{\bar{\lambda}}{|z|}\right)^{n-\alpha}\right)\left[u(z)^{\frac{n+\alpha}{n-\alpha}}-u_{\bar{\lambda}}(z)^{\frac{n+\alpha}{n-\alpha}}\right] d z>0 .
\end{aligned}
$$

Consequently, there exists $\epsilon_{1} \in(0,1)$ such that

$$
\left(u-u_{\bar{\lambda}}\right)(y) \geq \frac{\epsilon_{1}}{|y|^{n-\alpha}} \quad \forall|y| \geq \bar{\lambda}+1 .
$$

By the above and the explicit formula for $u_{\lambda}$, there exists $0<\epsilon_{2}<\epsilon_{1}$ such that

$$
\left(u-u_{\lambda}\right)(y) \geq \frac{\epsilon_{1}}{|y|^{n-\alpha}}+\left(u_{\bar{\lambda}}-u_{\lambda}\right)(y) \geq \frac{\epsilon_{1}}{2|y|^{n-\alpha}} \quad \forall|y| \geq \bar{\lambda}+1, \bar{\lambda} \leq \lambda \leq \bar{\lambda}+\epsilon_{2} .
$$

Now, for $\epsilon \in\left(0, \epsilon_{2}\right)$ which we choose below, we have, for $\bar{\lambda} \leq \lambda \leq \bar{\lambda}+\epsilon$ and for $\lambda \leq|y| \leq \bar{\lambda}+1$,

$$
\begin{aligned}
\left(u-u_{\lambda}\right)(y)= & \int_{|z| \geq \lambda} K(0, \lambda ; y, z)\left[u(z)^{\frac{n+\alpha}{n-\alpha}}-u_{\lambda}(z)^{\frac{n+\alpha}{n-\alpha}}\right] d z \\
\geq & \int_{\lambda \leq|z| \leq \bar{\lambda}+1} K(0, \lambda ; y, z)\left[u(z)^{\frac{n+\alpha}{n-\alpha}}-u_{\lambda}(z)^{\frac{n+\alpha}{n-\alpha}}\right] d z \\
& +\int_{\bar{\lambda}+2 \leq|z| \leq \bar{\lambda}+3} K(0, \lambda ; y, z)\left[u(z)^{\frac{n+\alpha}{n-\alpha}}-u_{\lambda}(z)^{\frac{n+\alpha}{n-\alpha}}\right] d z \\
\geq & \int_{\lambda \leq|z| \leq \bar{\lambda}+1} K(0, \lambda ; y, z)\left[u_{\bar{\lambda}}(z)^{\frac{n+\alpha}{n-\alpha}}-u_{\lambda}(z)^{\frac{n+\alpha}{n-\alpha}}\right] d z \\
& +\int_{\bar{\lambda}+2 \leq|z| \leq \bar{\lambda}+3} K(0, \lambda ; y, z)\left[u(z)^{\frac{n+\alpha}{n-\alpha}}-u_{\lambda}(z)^{\frac{n+\alpha}{n-\alpha}}\right] d z .
\end{aligned}
$$

Because of 40 , there exists $\delta_{1}>0$ such that

$$
u(z)^{\frac{n+\alpha}{n-\alpha}}-u_{\lambda}(z)^{\frac{n+\alpha}{n-\alpha}} \geq \delta_{1}, \quad \bar{\lambda}+2 \leq|z| \leq \bar{\lambda}+3 .
$$

Since

$$
K(0, \lambda ; y, z)=0, \quad \forall|y|=\lambda,
$$

$\left.\nabla_{y} K(0, \lambda ; y, z) \cdot y\right|_{|y|=\lambda}=(n-\alpha)|y-z|^{\alpha-n-2}\left(|z|^{2}-|y|^{2}\right)>0, \quad \forall \bar{\lambda}+2 \leq|z| \leq \bar{\lambda}+3$, and the function is smooth in the relevant region, we have, using also the positivity of the kernel,

$$
K(0, \lambda ; y, z) \geq \delta_{2}(|y|-\lambda), \quad \forall \bar{\lambda} \leq \lambda \leq|y| \leq \bar{\lambda}+1, \bar{\lambda}+2 \leq|z| \leq \bar{\lambda}+3,
$$


where $\delta_{2}>0$ is some constant independent of $\epsilon$. It is easy to see that for some constant $C>0$ independent of $\epsilon$, we have, for $\bar{\lambda} \leq \lambda \leq \bar{\lambda}+\epsilon$,

$$
\left|u_{\bar{\lambda}}(z)^{\frac{n+\alpha}{n-\alpha}}-u_{\lambda}(z)^{\frac{n+\alpha}{n-\alpha}}\right| \leq C(\lambda-\bar{\lambda}) \leq C \epsilon, \quad \forall \bar{\lambda} \leq \lambda \leq|z| \leq \bar{\lambda}+1,
$$

and (recall that $\lambda \leq|y| \leq \bar{\lambda}+1$ )

$$
\begin{aligned}
\int_{\lambda \leq|z| \leq \bar{\lambda}+1} K(0, \lambda ; y, z) d z \leq & \left|\int_{\lambda \leq|z| \leq \bar{\lambda}+1}\left(\frac{1}{|y-z|^{n-\alpha}}-\frac{1}{\left|y^{\lambda}-z\right|^{n-\alpha}}\right) d z\right| \\
& +\int_{\lambda \leq|z| \leq \bar{\lambda}+1}\left|\left(\frac{\lambda}{|y|}\right)^{n-\alpha}-1\right| \frac{1}{\left|y^{\lambda}-z\right|^{n-\alpha}} d z \\
\leq & C\left|y^{\lambda}-y\right|+C(|y|-\lambda) \leq C(|y|-\lambda) .
\end{aligned}
$$

It follows from the above that for small $\epsilon>0$ we have, for $\bar{\lambda} \leq \lambda \leq \bar{\lambda}+\epsilon$ and $\lambda \leq|y| \leq$ $\bar{\lambda}+1$,

$$
\begin{aligned}
\left(u-u_{\lambda}\right)(y) & \geq-C \epsilon \int_{\lambda \leq|z| \leq \bar{\lambda}+1} K(0, \lambda ; y, z) d z+\delta_{1} \delta_{2}(|y|-\lambda) \int_{\bar{\lambda}+2 \leq|z| \leq \bar{\lambda}+3} d z \\
& \geq\left(\delta_{1} \delta_{2} \int_{\bar{\lambda}+2 \leq|z| \leq \bar{\lambda}+3} d z-C \epsilon\right)(|y|-\lambda) \geq 0 .
\end{aligned}
$$

This and (40) violate the definition of $\bar{\lambda}$. Lemma 3.2 is established.

By the definition of $\bar{\lambda}(x)$,

$$
u_{x, \lambda}(y) \leq u(y), \quad \forall 0<\lambda<\bar{\lambda}(x),|y-x| \geq \lambda .
$$

Multiplying the above by $|y|^{n-\alpha}$ and sending $|y|$ to infinity yields

$$
\beta=\liminf _{|y| \rightarrow \infty}|y|^{n-\alpha} u(y) \geq \lambda^{n-\alpha} u(x), \quad \forall 0<\lambda<\bar{\lambda}(x) .
$$

On the other hand, if $\bar{\lambda}(\bar{x})<\infty$, we use Lemma 3.2 and multiply 38 by $|y|^{n-\alpha}$ and then send $|y|$ to infinity to obtain

$$
\beta=\lim _{|y| \rightarrow \infty}|y|^{n-\alpha} u(y)=\bar{\lambda}(\bar{x})^{n-\alpha} u(\bar{x})<\infty .
$$

Proof of Theorem 1.1. (i) If there exists some $\bar{x} \in \mathbb{R}^{n}$ such that $\bar{\lambda}(\bar{x})<\infty$, then, by 42 and 41, $\bar{\lambda}(x)<\infty$ for all $x \in \mathbb{R}^{n}$. Applying Lemma 3.2, we have

$$
u_{x, \bar{\lambda}(x)} \equiv u \quad \text { on } \mathbb{R}^{n}, \forall x \in \mathbb{R}^{n} .
$$

By a calculus lemma (Lemma 11.1 in [29], see also Lemma 2.5 in [30] for $\alpha=2$ ), any $C^{1}$ positive function $u$ satisfying the above must be of the form 5 .

(ii) If $\bar{\lambda}(x)=\infty$ for all $x \in \mathbb{R}^{n}$, then

$$
u_{x, \lambda}(y) \leq u(y), \quad \forall|y-x| \geq \lambda>0, x \in \mathbb{R}^{n} .
$$

By another calculus lemma (Lemma 11.2 in [29], see also Lemma 2.2 in [30] for $\alpha=2$ ), $u \equiv$ const, violating (4). Theorem 1.1 is established. 


\section{Proof of Theorem 1.4}

In this section we establish Theorem 1.4 .

Lemma 4.1. For $n \geq 1,0<\alpha<n$ and $\mu>0$, let $u$ be a Lebesgue measurable positive solution of (11) which is not identically equal to $\infty$. Then, for any $t<n /(n-\alpha)$ and $u \in L_{\text {loc }}^{\mu}\left(\mathbb{R}^{n}\right) \cap L_{\mathrm{loc}}^{t}\left(\mathbb{R}^{n}\right)$,

$$
\beta:=\liminf _{|x| \rightarrow \infty}|x|^{n-\alpha} u(x) \geq \int_{\mathbb{R}^{n}} u(y)^{\mu} d y>0,
$$

and

$$
\int_{|y|>2} \frac{u(y)^{\mu}}{|y|^{n-\alpha}} d y<\infty
$$

Proof. Multiplying 11 by $|x|^{n-\alpha}$, we obtain 43 by applying the Fatou lemma. Since $u$ is not identically equal to $\infty$, we see from (11) that $u$ is finite almost everywhere. So, for some $x_{1}, x_{2} \in B_{1}, x_{1} \neq x_{2}$, we have

$$
\sum_{i=1}^{2} \int_{\mathbb{R}^{n}} \frac{u(y)^{\mu}}{\left|x_{i}-y\right|^{n-\alpha}} d y \leq u\left(x_{1}\right)+u\left(x_{2}\right)<\infty .
$$

It follows that $u \in L_{\mathrm{loc}}^{\mu}\left(\mathbb{R}^{n}\right)$ and 44 holds. For $R>0$, we write

$$
u(x)=I_{R}(x)+I I_{R}(x):=\left(\int_{|y|<2 R}+\int_{|y|>2 R}\right) \frac{u(y)^{\mu}}{|x-y|^{n-\alpha}} d y .
$$

Since $u \in L_{\text {loc }}^{\mu}\left(\mathbb{R}^{n}\right)$ and 44 holds, $I I_{R} \in L^{\infty}\left(B_{R}\right)$. On the other hand, for any $1<t<$ $n /(n-\alpha)$, we have, by the Cauchy-Schwarz inequality,

$$
\begin{aligned}
\left\|I_{R}\right\|_{L^{t}\left(B_{R}\right)} & \leq \int_{|y|<2 R} u(y)^{\mu}\left\||\cdot-y|^{\alpha-n}\right\|_{L^{t}\left(B_{R}\right)} d y \\
& \leq\left\||\cdot-y|^{\alpha-n}\right\|_{L^{t}\left(B_{3 R}\right)} \int_{|y|<2 R} u(y)^{\mu} d y<\infty .
\end{aligned}
$$

Since $R>0$ is arbitrary, $u \in L_{\text {loc }}^{t}\left(\mathbb{R}^{n}\right)$.

Lemma 4.2. Assume $n \geq 1$ and $0<\alpha<n$.

(i) For $0<\mu<n /(n-\alpha)$, let $u$ be a positive Lebesgue measurable solution of (11) which is not identically infinity. Then $u \in C^{\infty}\left(\mathbb{R}^{n}\right)$.

(ii) For $\mu \geq n /(n-\alpha)$, let $u \in L_{\text {loc }}^{n(\mu-1) / \alpha}\left(\mathbb{R}^{n}\right)$ be a positive solution of 111 . Then $u \in C^{\infty}\left(\mathbb{R}^{n}\right)$.

Proof. (i) For $0<\mu<n /(n-2)$. We know from Lemma 4.1 that $u \in L_{\text {loc }}^{t}\left(\mathbb{R}^{n}\right)$ for all $t<n /(n-\alpha)$. For any $R>0$, write $u$ as in 45$)$. As usual, $I I_{R} \in C^{\infty}\left(B_{R}\right)$. For any $1<p<n / \mu(n-\alpha)$, let $1 / q=1 / p-\alpha / n$. Then $q>n /(n-\alpha)$. By the property of the Riesz potential,

$$
\left\|I_{R}\right\|_{L^{q}\left(B_{R}\right)} \leq C\left\|u^{\mu}\right\|_{L^{p}\left(B_{2 R}\right)}=C\|u\|_{L^{p \mu}\left(B_{2 R}\right)}^{\mu}<\infty .
$$


So $u \in L_{\mathrm{loc}}^{q}\left(\mathbb{R}^{n}\right)$. Let $\mu^{\prime}=\max (1, \mu)$. Since $u^{\mu} \leq C+C u^{\mu^{\prime}}$, we have

$$
u(x) \leq C \int_{|y|<2 R} \frac{V(y) u(y)}{|x-y|^{n-\alpha}} d y+h(x), \quad x \in B_{R},
$$

where

$$
V(y)=u(y)^{\mu^{\prime}-1}, \quad h(x)=C+\int_{|y|>2 R} \frac{u(y)^{\mu}}{|x-y|^{n-\alpha}} d y .
$$

By $44,, h \in L^{\infty}\left(B_{R}\right)$. Since $n\left(\mu^{\prime}-1\right) / \alpha<n /(n-\alpha)$, we obtain $V \in L_{\text {loc }}^{n / \alpha}\left(\mathbb{R}^{n}\right)$. Since $u \in L_{\text {loc }}^{q}\left(\mathbb{R}^{n}\right)$ with $q>n /(n-\alpha)$, we have, by applying Corollary $1.1, u \in L^{v}\left(B_{\epsilon(v)}\right)$ for any $v>0$, where $\epsilon(v)>0$. Now, back to 45 , $I_{R}$ is $C^{\infty}$ near the origin by bootstrapping. By the translation invariance of the problem, $u \in C^{\infty}\left(\mathbb{R}^{n}\right)$.

(ii) For $\mu \geq n /(n-\alpha)$, let $V(y)=u(y)^{\mu-1}$. We know from Lemma 4.1 that $u \in$ $L_{\mathrm{loc}}^{t}\left(\mathbb{R}^{n}\right)$ for all $t<n /(n-\alpha)$. Since $u \in L_{\mathrm{loc}}^{n(\mu-1) / \alpha}\left(\mathbb{R}^{n}\right)$ by assumption, we also have $V \in L_{\text {loc }}^{n / \alpha}\left(\mathbb{R}^{n}\right)$. Now, for any $R_{2}>R_{1}>0$, let

$$
h(y)=\int_{|y|>R_{2}} \frac{u(y)^{\mu}}{|x-y|^{n-\mu}} d y .
$$

Then $u \in L^{r}\left(B_{R_{2}}\right)$ with $r=n(\mu-1) / \alpha, V \in L^{n / \alpha}\left(B_{R_{2}}\right), h \in L^{\infty}\left(B_{R_{1}}\right) \subset L^{\nu}\left(B_{R_{1}}\right)$ for any $v>r$, and

$$
u(x)=\int_{|y|>R_{2}} \frac{V(y) u(y)}{|x-y|^{n-\alpha}} d y+h(x), \quad x \in B_{R_{1}} .
$$

By Corollary 1.1. $u \in L^{r}\left(B_{R_{1}}\right)$. Since $R_{1}>0$ is arbitrary, $u \in L_{\mathrm{loc}}^{r}\left(\mathbb{R}^{n}\right)$ for all $r>1$. Bootstrap as usual to get $u \in C^{\infty}\left(\mathbb{R}^{n}\right)$.

For $x \in \mathbb{R}^{n}, \lambda>0$, and a positive function $v$ on $\mathbb{R}^{n}$, let $v_{x, \lambda}$ be as in 6 .

Lemma 4.3. For $n \geq 1,0<\alpha<n$ and $\mu>0$, let $u$ be a positive solution of (11). Then

$$
u_{x, \lambda}(\xi)=\int_{\mathbb{R}^{n}} \frac{u_{x, \lambda}(z)^{\mu}}{|\xi-z|^{n-\alpha}}\left(\frac{\lambda}{|z-x|}\right)^{n+\alpha-\mu(n-\alpha)} d z, \quad \forall \xi \in \mathbb{R}^{n},
$$

and

$$
u(\xi)-u_{x, \lambda}(\xi)=\int_{|z-x| \geq \lambda} K(x, \lambda ; \xi, z)\left[u(z)^{\mu}-\left(\frac{\lambda}{|z-x|}\right)^{n+\alpha-\mu(n-\alpha)} u_{x, \lambda}(z)^{\mu}\right] d z,
$$

where

Moreover,

$$
K(x, \lambda ; \xi, z)=\frac{1}{|\xi-z|^{n-\alpha}}-\left(\frac{\lambda}{|\xi-x|}\right)^{n-\alpha} \frac{1}{\left|\xi^{x, \lambda}-z\right|^{n-\alpha}} .
$$

$$
K(x, \lambda ; \xi, z)>0, \quad \forall|\xi-x|,|z-x|>\lambda>0 .
$$

Proof. For $\mu=(n+\alpha) /(n-\alpha)$ the assertion is established in Section 3. The proof works for all $\mu>0$ with minor modifications. 
Lemma 4.4. For $n \geq 1,0<\alpha<n$ and $\mu>0$, let $u \in C^{1}\left(\mathbb{R}^{n}\right)$ be a positive solution of (11). Then for any $x \in \mathbb{R}^{n}$, there exists $\lambda_{0}(x)>0$ such that

$$
u_{x, \lambda}(y) \leq u(y), \quad \forall 0<\lambda<\lambda_{0}(x),|y-x| \geq \lambda .
$$

Proof. This has been proved in Section 3 for $\mu=(n+\alpha) /(n-\alpha)$. The same proof applies for all $\mu>0$.

Define, for $x \in \mathbb{R}^{n}$,

$$
\bar{\lambda}(x)=\sup \left\{\mu^{\prime}>0\left|u_{x, \lambda}(y) \leq u(y), \forall 0<\lambda<\mu^{\prime},\right| y-x \mid \geq \lambda\right\} .
$$

Lemma 4.5. For $n \geq 1,0<\alpha<n$ and $0<\mu<(n+\alpha) /(n-\alpha)$, let $u \in C^{1}\left(\mathbb{R}^{n}\right)$ be a positive solution of $\left[11\right.$. Then $\bar{\lambda}(x)=\infty$ for all $x \in \mathbb{R}^{n}$.

Proof. We argue by contradiction. Suppose that $\bar{\lambda}(\bar{x})<\infty$ for some $\bar{x} \in \mathbb{R}^{n}$. Without loss of generality, we may assume $\bar{x}=0$, and we write $\bar{\lambda}=\bar{\lambda}(0), u_{\lambda}=u_{0, \lambda}$. By the definition of $\bar{\lambda}$,

$$
u_{\bar{\lambda}}(y) \leq u(y), \quad \forall|y| \geq \bar{\lambda} .
$$

Since $n+\alpha-\mu(n-\alpha)>0,(\bar{\lambda} /|z|)^{n+\alpha-\mu(n-\alpha)}<1$ for $|z|>\bar{\lambda}$. So, by 49 and 477 with $x=0$ and $\lambda=\bar{\lambda}$, and the positivity of the kernel, we have, for $|y|>\bar{\lambda}$,

$$
\begin{aligned}
\left(u-u_{\bar{\lambda}}\right)(y) & =\int_{|z| \geq \bar{\lambda}} K(0, \bar{\lambda} ; y, z)\left[u(z)^{\mu}-\left(\frac{\lambda}{|z|}\right)^{n+\alpha-\mu(n-\alpha)} u_{\bar{\lambda}}(z)^{\mu}\right] d z \\
& \geq \int_{|z| \geq \bar{\lambda}} K(0, \bar{\lambda} ; y, z)\left[1-\left(\frac{\lambda}{|z|}\right)^{n+\alpha-\mu(n-\alpha)}\right] u_{\bar{\lambda}}(z)^{\mu} d z>0 .
\end{aligned}
$$

Thus, by the Fatou lemma and the above,

$$
\begin{aligned}
\liminf _{|y| \rightarrow \infty}|y|^{n-\alpha}\left(u-u_{\bar{\lambda}}\right)(y) & \geq \liminf _{|y| \rightarrow \infty} \int_{|z| \geq \bar{\lambda}}|y|^{n-\alpha} K(0, \bar{\lambda} ; y, z)\left[u(z)^{\mu}-u_{\bar{\lambda}}(z)^{\mu}\right] d z \\
& \geq \int_{|z| \geq \bar{\lambda}}\left(1-\left(\frac{\bar{\lambda}}{|z|}\right)^{n-\alpha}\right)\left[u(z)^{\mu}-u_{\bar{\lambda}}(z)^{\mu}\right] d z>0 .
\end{aligned}
$$

Consequently, there exists $\epsilon_{1} \in(0,1)$ such that

$$
\left(u-u_{\bar{\lambda}}\right)(y) \geq \frac{\epsilon_{1}}{|y|^{n-\alpha}}, \quad \forall|y| \geq \bar{\lambda}+1 .
$$

By the above and the explicit formula for $u_{\lambda}$, there exists $0<\epsilon_{2}<\epsilon_{1}$ such that

$$
\left(u-u_{\lambda}\right)(y) \geq \frac{\epsilon_{1}}{|y|^{n-\alpha}}+\left(u_{\bar{\lambda}}-u_{\lambda}\right)(y) \geq \frac{\epsilon_{1}}{2|y|^{n-\alpha}} \quad \forall|y| \geq \bar{\lambda}+1, \bar{\lambda} \leq \lambda \leq \bar{\lambda}+\epsilon_{2} .
$$

Now, using (49) and (50) as in Section 3, for $\epsilon \in\left(0, \epsilon_{2}\right)$ which we choose below, we have, for $\bar{\lambda} \leq \lambda \leq \bar{\lambda}+\epsilon$ and for $\lambda \leq|y| \leq \bar{\lambda}+1$,

$$
\begin{aligned}
\left(u-u_{\lambda}\right)(y) \geq & \int_{\lambda \leq|z| \leq \bar{\lambda}+1} K(0, \lambda ; y, z)\left[u_{\bar{\lambda}}(z)^{\mu}-u_{\lambda}(z)^{\mu}\right] d z \\
& +\int_{\bar{\lambda}+2 \leq|z| \leq \bar{\lambda}+3} K(0, \lambda ; y, z)\left[u(z)^{\mu}-u_{\lambda}(z)^{\mu}\right] d z .
\end{aligned}
$$


Because of (50), there exists $\delta_{1}>0$ such that

$$
u(z)^{\mu}-u_{\lambda}(z)^{\mu} \geq \delta_{1}, \quad \bar{\lambda}+2 \leq|z| \leq \bar{\lambda}+3 .
$$

It was shown in Section 3 that

$$
K(0, \lambda ; y, z) \geq \delta_{2}(|y|-\lambda), \quad \forall \bar{\lambda} \leq \lambda \leq|y| \leq \bar{\lambda}+1, \bar{\lambda}+2 \leq|z| \leq \bar{\lambda}+3,
$$

where $\delta_{2}>0$ is some constant independent of $\epsilon$. It is easy to see that for some constant $C>0$ independent of $\epsilon$, we have, for $\bar{\lambda} \leq \lambda \leq \bar{\lambda}+\epsilon$,

$$
\left|u_{\bar{\lambda}}(z)^{\mu}-u_{\lambda}(z)^{\mu}\right| \leq C(\lambda-\bar{\lambda}) \leq C \epsilon, \quad \forall \bar{\lambda} \leq \lambda \leq|z| \leq \bar{\lambda}+1,
$$

and (recall that $\lambda \leq|y| \leq \bar{\lambda}+1$ ), as in Section 3,

$$
\int_{\lambda \leq|z| \leq \bar{\lambda}+1} K(0, \lambda ; y, z) d z \leq C(|y|-\lambda)
$$

It follows from the above that for small $\epsilon>0$ we have, for $\bar{\lambda} \leq \lambda \leq \bar{\lambda}+\epsilon$ and $\lambda \leq|y| \leq$ $\bar{\lambda}+1$,

$$
\begin{aligned}
\left(u-u_{\lambda}\right)(y) & \geq-C \epsilon \int_{\lambda \leq|z| \leq \bar{\lambda}+1} K(0, \lambda ; y, z) d z+\delta_{1} \delta_{2}(|y|-\lambda) \int_{\bar{\lambda}+2 \leq|z| \leq \bar{\lambda}+3} d z \\
& \geq\left(\delta_{1} \delta_{2} \int_{\bar{\lambda}+2 \leq|z| \leq \bar{\lambda}+3} d z-C \epsilon\right)(|y|-\lambda) \geq 0 .
\end{aligned}
$$

This and (64) violate the definition of $\bar{\lambda}$. Lemma 4.5 is established.

Proof of Theorem 1.4. According to Lemma $4.5, \bar{\lambda}(x)=\infty$ for all $x \in \mathbb{R}^{n}$, i.e.,

$$
u_{x, \lambda}(y) \leq u(y), \quad \forall|y-x| \geq \lambda>0, x \in \mathbb{R}^{n} .
$$

By a calculus lemma (Lemma 11.2 in [29], see also Lemma 2.2 in [30] for $\alpha=2$ ), $u \equiv$ const, violating [11]. Theorem 1.4 is established.

\section{Proof of Theorem 1.5}

In this section we establish Theorem 1.5 ,

Lemma 5.1. For $n \geq 1$ and $p, q>0$, let $u$ be a nonnegative Lebesgue measurable function in $\mathbb{R}^{n}$ satisfying $[12$. Then

$$
\begin{gathered}
\int_{\mathbb{R}^{n}}\left(1+|y|^{p}\right) u(y)^{-q} d y<\infty, \\
\gamma:=\lim _{|x| \rightarrow \infty}|x|^{-p} u(x) \\
=\lim _{|x| \rightarrow \infty} \int_{\mathbb{R}^{n}} \frac{|x-y|^{p}}{|x|^{p}} u(y)^{-q} d y \int_{\mathbb{R}^{n}} u(y)^{-q} d y \in(0, \infty),
\end{gathered}
$$

and, for some constant $C \geq 1$,

$$
\frac{1+|x|^{p}}{C} \leq u(x) \leq C\left(1+|x|^{p}\right), \quad \forall x \in \mathbb{R}^{n} .
$$


Proof. We see from (12) that $u$ must be positive everywhere and

$$
\left|\left\{y \in \mathbb{R}^{n} \mid u(y)<\infty\right\}\right|>0,
$$

where $|\cdot|$ denotes the Lebesgue measure of the set. So there exist $R>1$ and some measurable set $E$ such that

$$
E \subset\{y \mid u(y)<R\} \cap B_{R}, \quad|E| \geq 1 / R .
$$

By (12),

$$
\begin{aligned}
u(x) & =\int_{\mathbb{R}^{n}}|x-y|^{p} u(y)^{-q} d y \geq \int_{E}|x-y|^{p} u(y)^{-q} d y \\
& \geq R^{-q} \int_{E}|x-y|^{p} d y, \quad \forall x \in \mathbb{R}^{n} .
\end{aligned}
$$

The first inequality in 53 follows from the above.

For some $1 \leq|\bar{x}| \leq 2$,

$$
\int_{\mathbb{R}^{n}}|\bar{x}-y|^{p} u(y)^{-q} d y=u(\bar{x})<\infty .
$$

We deduce 51 from the first inequality in $(53)$ and the above.

For $|x| \geq 1$,

$$
\left|\frac{|x-y|^{p}}{|x|^{p}} u(y)^{-q}\right| \leq\left(1+|y|^{p}\right) u(y)^{-q},
$$

so, in view of (51), 52 follows from the Lebesgue dominated convergence theorem. The second inequality in (53) follows from (12), 51) and (52).

Lemma 5.2. For $n \geq 1$ and $p, q>0$, let $u$ be a nonnegative Lebesgue measurable function in $\mathbb{R}^{n}$ satisfying 12 , Then $u \in C^{\infty}\left(\mathbb{R}^{n}\right)$.

Proof. For $R>0$, write (12) as

$$
u(x)=I_{R}(x)+I I_{R}(x):=\left(\int_{|y| \leq 2 R}+\int_{|y|>2 R}\right)|x-y|^{p} u(y)^{-q} d y .
$$

Because of (51), we can differentiate $I I_{R}(x)$ under the integral sign for $|x|<R$, and therefore $I I_{R} \in C^{\infty}\left(B_{R}\right)$. On the other hand, since $u^{-q} \in L^{\infty}\left(B_{2 R}\right)$, clearly $I_{R}$ is at least Hölder continuous in $B_{R}$. Since $R>0$ is arbitrary, $u$ is Hölder continuous in $\mathbb{R}^{n}$. Now $u^{-q}$ is Hölder continuous in $B_{2 R}$, the regularity of $I_{R}$ further improves and, by bootstrap, we eventually have $u \in C^{\infty}\left(\mathbb{R}^{n}\right)$.

Let $v$ be a positive function on $\mathbb{R}^{n}$. For $x \in \mathbb{R}^{n}$ and $\lambda>0$, consider

$$
v_{x, \lambda}(\xi)=\left(\frac{|\xi-x|}{\lambda}\right)^{p} v\left(\xi^{x, \lambda}\right), \quad \xi \in \mathbb{R}^{n}
$$

where

$$
\xi^{x, \lambda}=x+\frac{\lambda^{2}(\xi-x)}{|\xi-x|^{2}} .
$$

Note that the notation $v_{x, \lambda}$ in this section is different from that in Sections 1-4. 
Making a change of variables

$$
y=z^{x, \lambda}=x+\frac{\lambda^{2}(z-x)}{|z-x|^{2}}
$$

we have

$$
d y=\left(\frac{\lambda}{|z-x|}\right)^{2 n} d z
$$

Thus

$$
\begin{aligned}
\int_{|y-x| \geq \lambda}\left|\xi^{x, \lambda}-y\right|^{p} v(y)^{-q} d y & =\int_{|z-x| \leq \lambda}\left|\xi^{x, \lambda}-z^{x, \lambda}\right|^{p} v\left(z^{x, \lambda}\right)^{-q}\left(\frac{\lambda}{|z-x|}\right)^{2 n} d z \\
& =\int_{|z-x| \leq \lambda}\left|\xi^{x, \lambda}-z^{x, \lambda}\right|^{p}\left(\frac{\lambda}{|z-x|}\right)^{2 n-p q} v_{x, \lambda}(z)^{-q} d z
\end{aligned}
$$

Using (30), we have

$$
\begin{aligned}
& \left(\frac{\lambda}{|\xi-x|}\right)^{-p} \int_{|y-x| \geq \lambda}\left|\xi^{x, \lambda}-y\right|^{p} v(y)^{-q} d y \\
& =\int_{|z-x| \leq \lambda}|\xi-z|^{p}\left(\frac{\lambda}{|z-x|}\right)^{2 n-p q+p} v_{x, \lambda}(z)^{-q} d z .
\end{aligned}
$$

Similarly,

$$
\begin{aligned}
& \left(\frac{\lambda}{|\xi-x|}\right)^{-p} \int_{|y-x| \leq \lambda}\left|\xi^{x, \lambda}-y\right|^{p} v(y)^{-q} d y \\
& =\int_{|z-x| \geq \lambda}|\xi-z|^{p}\left(\frac{\lambda}{|z-x|}\right)^{2 n-p q+p} v_{x, \lambda}(z)^{-q} d z .
\end{aligned}
$$

Lemma 5.3. Let $u$ be a positive solution of (12). Then

$$
u_{x, \lambda}(\xi)=\int_{\mathbb{R}^{n}}|\xi-z|^{p}\left(\frac{\lambda}{|z-x|}\right)^{2 n-p q+p} u_{x, \lambda}(z)^{-q} d z, \quad \forall \xi \in \mathbb{R}^{n},
$$

and

$$
u_{x, \lambda}(\xi)-u(\xi)=\int_{|z-x| \geq \lambda} k(x, \lambda ; \xi, z)\left[u(z)^{-q}-\left(\frac{\lambda}{|z-x|}\right)^{2 n-p q+p} u_{x, \lambda}(z)^{-q}\right] d z,
$$

where

$$
k(x, \lambda ; \xi, z)=\left(\frac{|\xi-x|}{\lambda}\right)^{p}\left|\xi^{x, \lambda}-z\right|^{p}-|\xi-z|^{p}
$$

Moreover

$$
k(x, \lambda ; \xi, z)>0, \quad \forall|\xi-x|,|z-x|>\lambda>0 .
$$


Proof. Since $\left(\xi^{x, \lambda}\right)^{x, \lambda}=\xi$ and $\left(v_{x, \lambda}\right)_{x, \lambda} \equiv v$, identity 56 follows from 12 and 54 and 55 with $v=u$. Similarly, using also (56), we obtain

$$
\begin{aligned}
u(\xi)= & \int_{|z-x| \geq \lambda}|\xi-z|^{p} u(z)^{-q} d z+\int_{|y-x|<\lambda}|\xi-y|^{p} u(y)^{-q} d y \\
= & \int_{|z-x| \geq \lambda}|\xi-z|^{p} u(z)^{-q} d z \\
& +\left(\frac{|\xi-x|}{\lambda}\right)^{p} \int_{|z-x| \geq \lambda}\left|\xi^{x, \lambda}-z\right|^{p}\left(\frac{\lambda}{|z-x|}\right)^{2 n-p q+p} u_{x, \lambda}(z)^{-q} d z \\
u_{x, \lambda}(\xi)= & \int_{\mathbb{R}^{n}}|\xi-z|^{p}\left(\frac{\lambda}{|z-x|}\right)^{2 n-p q+p} u_{x, \lambda}(z)^{-q} d z \\
= & \int_{|z-x| \geq \lambda}|\xi-z|^{p}\left(\frac{\lambda}{|z-x|}\right)^{2 n-p q+p} u_{x, \lambda}(z)^{-q} d z \\
& +\left(\frac{|\xi-x|}{\lambda}\right)^{p} \int_{|z-x| \geq \lambda}\left|\xi^{x, \lambda}-z\right|^{p} u(z)^{-q} d z .
\end{aligned}
$$

Identity (57) follows from the above. The positivity of the kernel $k$ is elementary.

Lemma 5.4. For $n \geq 1$ and $p, q>0$, let $u$ be a solution of 12 . Then for any $x \in \mathbb{R}^{n}$, there exists $\lambda_{0}(x)>0$ such that

$$
u_{x, \lambda}(y) \geq u(y), \quad \forall 0<\lambda<\lambda_{0}(x),|y-x| \geq \lambda .
$$

Proof. The proof is similar to that of Lemma 2.1 in [29] and Lemma 3.1 in Section 3. Without loss of generality we may assume $x=0$, and we write $u_{\lambda}=u_{0, \lambda}$.

Since $p>0$ and $u$ is a positive $C^{1}$ function, there exists $r_{0}>0$ such that

$$
\nabla_{y}\left(|y|^{-p / 2} u(y)\right) \cdot y<0, \quad \forall 0<|y|<r_{0} .
$$

Consequently,

$$
u_{\lambda}(y)>u(y), \quad \forall 0<\lambda<|y|<r_{0} .
$$

By (53),

$$
u(z) \leq C\left(r_{0}\right)|z|^{p} \quad \forall|z| \geq r_{0} .
$$

For small $\lambda_{0} \in\left(0, r_{0}\right)$ and for $0<\lambda<\lambda_{0}$, we have, using (53) and [59),

$$
u_{\lambda}(y)=\left(\frac{|y|}{\lambda}\right)^{p} u\left(\frac{\lambda^{2} y}{|y|^{2}}\right) \geq\left(\frac{|y|}{\lambda_{0}}\right)^{p} \inf _{B_{r_{0}}} u \geq u(y), \quad \forall|y| \geq r_{0} .
$$

Estimate (58), with $x=0$ and $\lambda_{0}(x)=\lambda_{0}$, follows from (59) and the above.

Define, for $x \in \mathbb{R}^{n}$,

$$
\bar{\lambda}(x)=\sup \left\{\mu>0\left|u_{x, \lambda}(y) \geq u(y), \forall 0<\lambda<\mu,\right| y-x \mid \geq \lambda\right\} .
$$


Lemma 5.5. For $n \geq 1, p>0$ and $0<q \leq 1+2 n / p$, let $u$ be $a$ solution of (12). Then

$$
\bar{\lambda}(x)<\infty, \quad \forall x \in \mathbb{R}^{n},
$$

and

$$
u_{x, \lambda(x)} \equiv u \quad \text { on } \mathbb{R}^{n}, \forall x \in \mathbb{R}^{n} .
$$

Consequently, $q=1+2 n / p$.

Proof. By the definition of $\bar{\lambda}(x)$,

$$
u_{x, \lambda}(y) \geq u(y), \quad \forall 0<\lambda<\bar{\lambda}(x),|y-x| \geq \lambda .
$$

Multiplying the above by $|y|^{-p}$ and sending $|y|$ to infinity yields, using [52),

$$
0<\gamma=\lim _{|y| \rightarrow \infty}|y|^{-p} u(y) \leq \lambda^{-p} u(x), \quad \forall 0<\lambda<\bar{\lambda}(x) .
$$

Thus $\bar{\lambda}(x)<\infty$ for all $x \in \mathbb{R}^{n}$.

Now we prove (61). Without loss of generality, we may assume $x=0$, and we write $\bar{\lambda}=\bar{\lambda}(0), u_{\lambda}=u_{0, \lambda}$, and $y^{\lambda}=y^{0, \lambda}$. By the definition of $\bar{\lambda}$,

$$
u_{\bar{\lambda}}(y) \geq u(y), \quad \forall|y| \geq \bar{\lambda} .
$$

Since $2 n-p q+p \geq 0$, we have $(\bar{\lambda} /|z|)^{2 n-p q+p} \leq 1$ for $|z| \geq \bar{\lambda}$. So, by 63, , 57, with $x=0$ and $\lambda=\bar{\lambda}$, and the positivity of the kernel, either $u_{\bar{\lambda}}(y)=u(y)$ for all $|y| \geq \bar{\lambda}-$ then we are done (using (57) to see that $2 n-p q+p=0$ )—or $u_{\bar{\lambda}}(y)>u(y)$ for all $|y|>\bar{\lambda}$, which we assume below.

By 57, with $x=0$ and $\lambda=\bar{\lambda}$, and the Fatou lemma,

$$
\begin{aligned}
\liminf _{|y| \rightarrow \infty}|y|^{-p}\left(u_{\bar{\lambda}}-u\right)(y) & \\
\quad= & \liminf _{|y| \rightarrow \infty} \int_{|z| \geq \bar{\lambda}}|y|^{-p} k(0, \bar{\lambda} ; y, z)\left[u(z)^{-q}-\left(\frac{\bar{\lambda}}{|z|}\right)^{2 n-p q+p} u_{\bar{\lambda}}(z)^{-q}\right] d z \\
& \geq \int_{|z| \geq \bar{\lambda}}\left(\left(\frac{|z|}{\bar{\lambda}}\right)^{p}-1\right)\left[u(z)^{-q}-u_{\bar{\lambda}}(z)^{-q}\right] d z>0 .
\end{aligned}
$$

Consequently, using also the positivity of $u_{\bar{\lambda}}-u$, there exists $\epsilon_{1} \in(0,1)$ such that

$$
\left(u_{\bar{\lambda}}-u\right)(y) \geq \epsilon_{1}|y|^{p}, \quad \forall|y| \geq \bar{\lambda}+1 .
$$

By the above and the explicit formula of $u_{\lambda}$, there exists $0<\epsilon_{2}<\epsilon_{1}$ such that

$$
\left(u_{\lambda}-u\right)(y) \geq \epsilon_{1}|y|^{p}+\left(u_{\lambda}-u_{\bar{\lambda}}\right)(y) \geq \frac{\epsilon_{1}}{2}|y|^{p}, \quad \forall|y| \geq \bar{\lambda}+1, \bar{\lambda} \leq \lambda \leq \bar{\lambda}+\epsilon_{2} .
$$


Recall that $2 n-p q+p \geq 0$ and therefore $(\lambda /|z|)^{2 n-p q+p} \leq 1$ for $|z| \geq \lambda$. For $\epsilon \in\left(0, \epsilon_{2}\right)$ which we choose below, we have, for $\bar{\lambda} \leq \lambda \leq \bar{\lambda}+\epsilon$ and for $\lambda \leq|y| \leq \bar{\lambda}+1$,

$$
\begin{aligned}
\left(u_{\lambda}-u\right)(y) \geq & \int_{|z| \geq \lambda} k(0, \lambda ; y, z)\left[u(z)^{-q}-u_{\lambda}(z)^{-q}\right] d z \\
\geq & \int_{\lambda \leq|z| \leq \bar{\lambda}+1} k(0, \lambda ; y, z)\left[u(z)^{-q}-u_{\lambda}(z)^{-q}\right] d z \\
& +\int_{\bar{\lambda}+2 \leq|z| \leq \bar{\lambda}+3} k(0, \lambda ; y, z)\left[u(z)^{-q}-u_{\lambda}(z)^{-q}\right] d z \\
\geq & \int_{\lambda \leq|z| \leq \bar{\lambda}+1} k(0, \lambda ; y, z)\left[u_{\bar{\lambda}}(z)^{-q}-u_{\lambda}(z)^{-q}\right] d z \\
& +\int_{\bar{\lambda}+2 \leq|z| \leq \bar{\lambda}+3} k(0, \lambda ; y, z)\left[u(z)^{-q}-u_{\lambda}(z)^{-q}\right] d z .
\end{aligned}
$$

Because of 64, there exists $\delta_{1}>0$ such that

$$
u(z)^{-q}-u_{\lambda}(z)^{-q} \geq \delta_{1}, \quad \bar{\lambda}+2 \leq|z| \leq \bar{\lambda}+3 .
$$

Since

$$
\begin{gathered}
k(0, \lambda ; y, z)=0, \quad \forall|y|=\lambda, \\
\left.\nabla_{y} k(0, \lambda ; y, z) \cdot y\right|_{|y|=\lambda}=p|y-z|^{p-2}\left(|z|^{2}-|y|^{2}\right)>0, \quad \forall \bar{\lambda}+2 \leq|z| \leq \bar{\lambda}+3,
\end{gathered}
$$

and the function is smooth in the relevant region, we have, using also the positivity of the kernel,

$$
k(0, \lambda ; y, z) \geq \delta_{2}(|y|-\lambda), \quad \forall \bar{\lambda} \leq \lambda \leq|y| \leq \bar{\lambda}+1, \bar{\lambda}+2 \leq|z| \leq \bar{\lambda}+3,
$$

where $\delta_{2}>0$ is some constant independent of $\epsilon$. It is easy to see that for some constant $C>0$ independent of $\epsilon$, we have, for $\bar{\lambda} \leq \lambda \leq \bar{\lambda}+\epsilon$,

$$
\left|u_{\bar{\lambda}}(z)^{-q}-u_{\lambda}(z)^{-q}\right| \leq C(\lambda-\bar{\lambda}) \leq C \epsilon, \quad \forall \bar{\lambda} \leq \lambda \leq|z| \leq \bar{\lambda}+1,
$$

and (recall that $\lambda \leq|y| \leq \bar{\lambda}+1$ )

$$
\begin{aligned}
\int_{\lambda \leq|z| \leq \bar{\lambda}+1} k(0, \lambda ; y, z) d z & \leq C(|y|-\lambda)+\int_{\lambda \leq|z| \leq \bar{\lambda}+1}\left(\left|y^{\lambda}-z\right|^{p}-|y-z|^{p}\right) d z \\
& \leq C(|y|-\lambda)+C\left|y^{\lambda}-y\right| \leq C(|y|-\lambda) .
\end{aligned}
$$

It follows that for small $\epsilon>0$ we have, for $\bar{\lambda} \leq \lambda \leq \bar{\lambda}+\epsilon$ and $\lambda \leq|y| \leq \bar{\lambda}+1$,

$$
\begin{aligned}
\left(u_{\lambda}-u\right)(y) & \geq-C \epsilon \int_{\lambda \leq|z| \leq \bar{\lambda}+1} k(0, \lambda ; y, z) d z+\delta_{1} \delta_{2}(|y|-\lambda) \int_{\bar{\lambda}+2 \leq|z| \leq \bar{\lambda}+3} d z \\
& \geq\left(\delta_{1} \delta_{2} \int_{\bar{\lambda}+2 \leq|z| \leq \bar{\lambda}+3} d z-C \epsilon\right)(|y|-\lambda) \geq 0 .
\end{aligned}
$$

This and 64 violate the definition of $\bar{\lambda}$. Lemma 5.5 is established. 
Proof of Theorem 1.5. According to Lemma 5.5, $q=1+2 n / p$ and

$$
u_{x, \bar{\lambda}(x)} \equiv u \quad \text { on } \mathbb{R}^{n}, \forall x \in \mathbb{R}^{n} .
$$

By a calculus lemma (Lemma 11.1 in [29], see also Lemma 2.5 in [30] for $\alpha=2$ ), any $C^{1}$ positive function $u$ satisfying the above must be of the form 13 .

\section{Appendix A}

In this appendix, we show, as pointed out in Remark 1.2 that for some $a=a(n, p)>0$, (13) solves (12) with $q=1+2 n / p$. Our proof works equally well for equation (4).

For $n \geq 1$ and $p \in(-n, 0) \cup(0, \infty)$, we consider the integral equation

$$
u(x)=\int_{\mathbb{R}^{n}}|x-y|^{p} u(y)^{-(2 n+p) / p} d y, \quad \forall x \in \mathbb{R}^{n} .
$$

Lemma 5.6. For $n \geq 1$ and $p \in(-n, 0) \cup(0, \infty)$, there exists a unique $a=a(n, p)>0$ such that for any $\bar{x} \in \mathbb{R}^{n}$ and $d>0$,

$$
u(x)=\left(\frac{d+|x-\bar{x}|^{2}}{a}\right)^{p / 2}
$$

satisfies 65.

Proof. Let $q=1+2 n / p$. For a positive function $v$, and for $x \in \mathbb{R}^{n}$ and $\lambda>0$, we set

$$
v_{x, \lambda}(\xi)=\left(\frac{|\xi-x|}{\lambda}\right)^{p} v\left(\xi^{x, \lambda}\right), \quad \xi \in \mathbb{R}^{n},
$$

where $\xi^{x, \lambda}$ is given by 77 . By conformal invariance, we only need to prove that modulo a positive constant multiple,

$$
u(x):=\left(1+|x|^{2}\right)^{p / 2}
$$

satisfies 65. Set

$$
\tilde{u}(x)=\int_{\mathbb{R}^{n}}|x-y|^{p} u(y)^{-q} d y, \quad x \in \mathbb{R}^{n} .
$$

We only need to show that $\tilde{u}$ is a constant multiple of $u$.

For any $x \in \mathbb{R}^{n}$, let $\lambda(x):=\sqrt{1+|x|^{2}}$. Observe that

$$
u_{x, \lambda(x)} \equiv u, \quad \text { on } \mathbb{R}^{n}, \forall x \in \mathbb{R}^{n} .
$$

Making a change of variables

$$
y=z^{x, \lambda(x)}=x+\frac{\lambda(x)^{2}(z-x)}{|z-x|^{2}},
$$

we have, using (66) and the conformal invariance of the equation (54), 55, , 31) and (32),

$$
\begin{aligned}
\tilde{u}_{x, \lambda(x)}(\xi) & =\left(\frac{|\xi-x|}{\lambda(x)}\right)^{p} \int_{\mathbb{R}^{n}}\left|\xi^{x, \lambda(x)}-y\right|^{p} u(y)^{-q} d y \\
& =\int_{\mathbb{R}^{n}}|\xi-z|^{p} u_{x, \lambda(x)}(z)^{-q} d z=\tilde{u}(\xi) .
\end{aligned}
$$


Multiplying this by $|\xi|^{-p}$ and sending $|\xi|$ to $\infty$ leads to

$$
\lim _{|\xi| \rightarrow \infty}|\xi|^{-p} \tilde{u}(\xi)=\lambda(x)^{-p} \tilde{u}(x)=\left(1+|x|^{2}\right)^{-p / 2} \tilde{u}(x)=u(x)^{-1} \tilde{u}(x), \quad \forall x \in \mathbb{R}^{n} .
$$

So $\tilde{u}$ is a constant multiple of $u$, and we are done.

\section{Appendix B}

In this appendix, we present some calculus lemmas obtained jointly with L. Nirenberg. These lemmas under the stronger assumption $f \in C^{1}\left(\mathbb{R}^{n}\right)$ have been used repeatedly in some works on Liouville type theorems for conformally invariant equations (see, e.g., [29], [30], [24]-[27] and the present paper).

Lemma 5.7. For $n \geq 1$ and $v \in \mathbb{R}$, let $f$ be a function defined on $\mathbb{R}^{n}$ with values in $[-\infty, \infty]$ satisfying

$$
\left(\frac{\lambda}{|y-x|}\right)^{v} f\left(x+\frac{\lambda^{2}(y-x)}{|y-x|^{2}}\right) \leq f(y), \quad \forall|x-y|>\lambda>0 .
$$

Then $f \equiv$ const or $\pm \infty$.

Remark 5.1. If the first inequality in 67) is reversed, the conclusion still holds, since we can replace $f$ by $-f$.

Proof. For all $b>1$ and $y, z \in \mathbb{R}^{n}$ with $y \neq z$, let

$$
x=x(b)=y+b(z-y), \quad \lambda=\lambda(b)=\sqrt{|z-x||y-x|} .
$$

Then

and, by 67),

$$
z=x+\frac{\lambda^{2}(y-x)}{|y-x|^{2}}
$$

$$
\left(\frac{\lambda}{|y-x|}\right)^{v} f(z) \leq f(y)
$$

Since

$$
\lim _{b \rightarrow \infty} \frac{\lambda}{|y-x|}=\lim _{b \rightarrow \infty} \sqrt{\frac{|z-x|}{|y-x|}}=1,
$$

we have $f(z) \leq f(y)$. Lemma 5.7 follows since $y \neq z$ are arbitrary.

Lemma 5.8. Let $n \geq 1, v \in \mathbb{R}$ and $f \in C^{0}\left(\mathbb{R}^{n}\right)$. Suppose that for every $x \in \mathbb{R}^{n}$, there exists $\lambda(x)>0$ such that

$$
\left(\frac{\lambda(x)}{|y-x|}\right)^{v} f\left(x+\frac{\lambda(x)^{2}(y-x)}{|y-x|^{2}}\right)=f(y), \quad \forall y \in \mathbb{R}^{n} \backslash\{x\} .
$$

Then, for some $a \geq 0, d>0$ and $\bar{x} \in \mathbb{R}^{n}$,

$$
f(x) \equiv \pm a\left(\frac{1}{d+|x-\bar{x}|^{2}}\right)^{v / 2}
$$


Proof. By (68) and the continuity of $f$,

$$
\alpha:=\lim _{|y| \rightarrow \infty}|y|^{\nu} f(y)=\lambda(x)^{\nu} f(x), \quad \forall x \in \mathbb{R}^{n} .
$$

If $v=0$, then $f \equiv \alpha$, and we are done. On the other hand, the case $v<0$ can easily be reduced to the case of $v>0$ if we let $z=x+\lambda(x)^{2}(y-x) /|y-x|^{2}$ in 68 . So we will assume that $v>0$.

If $\alpha=0$, then $f \equiv 0$, and we are done. Otherwise, replacing $f$ by a nonzero multiple of $f$, we may assume that $\alpha=1$. Since $f(y) \rightarrow 0$ as $|y|$ tends to $\infty$, and since $f$ is continuous and positive, $f$ has a maximum point, and we may assume that $f$ has a maximum point at the origin.

For any $x \in \mathbb{R}^{n}$, we have, for large $|y|$,

$$
\begin{aligned}
|y|^{v} f(y) & =\lambda(x)^{v}\left(\frac{|y|}{|y-x|}\right)^{v} f\left(x+\frac{\lambda(x)^{2}(y-x)}{|y-x|^{2}}\right) \\
& =\lambda(x)^{v}\left[1+\frac{v x \cdot y}{|y|^{2}}+O\left(|y|^{-2}\right)\right] f\left(x+\frac{\lambda(x)^{2}(y-x)}{|y-x|^{2}}\right),
\end{aligned}
$$

and, by 69 and $\alpha=1$,

$$
\begin{aligned}
|y|\left[|y|^{v} f(y)-1\right]= & |y| \lambda(x)^{v}\left[f\left(x+\frac{\lambda(x)^{2}(y-x)}{|y-x|^{2}}\right)-f(x)\right] \\
& +\left[\frac{\lambda(x)^{v} v x \cdot y}{|y|}+O\left(|y|^{-1}\right)\right] f\left(x+\frac{\lambda(x)^{2}(y-x)}{|y-x|^{2}}\right) .
\end{aligned}
$$

Taking $x=0$ in the above and using the fact that $f$ has a maximum point at the origin, we obtain

$$
\limsup _{|y| \rightarrow \infty}|y|\left[|y|^{\nu} f(y)-1\right] \leq 0 .
$$

Claim. For any $\epsilon>0$, there exists $M_{\epsilon}$ such that for any $|y| \geq M_{\epsilon}$, there exists $\tilde{x}=\tilde{x}(y)$ satisfying

$$
\tilde{x}+\frac{\lambda(\tilde{x})^{2}(y-\tilde{x})}{|y-\tilde{x}|^{2}}=0 \quad \text { and } \quad|\tilde{x}| \leq \epsilon .
$$

Indeed, we know from 69) and $\alpha=1$ that $\lambda(x)=f(x)^{-1 / v}$ for all $x \in \mathbb{R}^{n}$. For any $\epsilon \in(0,1)$, pick $M_{\epsilon}>1$ so that

$$
\left(M_{\epsilon}-\epsilon\right)^{-1} \max _{|x| \leq \epsilon} f(x)^{-2 / v}<\frac{\epsilon}{2} .
$$

Then, for all $|y| \geq M_{\epsilon}$,

$$
\max _{|x| \leq \epsilon}\left|\frac{\lambda(x)^{2}(y-x)}{|y-x|^{2}}\right|=\max _{|x| \leq \epsilon}|y-x|^{-1} f(x)^{-2 / v} \leq\left(M_{\epsilon}-\epsilon\right)^{-1} \max _{|x| \leq \epsilon} f(x)^{-2 / v}<\frac{\epsilon}{2} .
$$

Thus, by a degree argument using the continuity of $f$, there exists $\tilde{x}=\tilde{x}(y)$ satisfying (72). 
With $x=\tilde{x}(y)$ in $(70)$, we obtain

$$
\liminf _{|y| \rightarrow \infty}|y|\left[|y|^{v} f(y)-1\right] \geq-\epsilon v f(0) \max _{|z| \leq \epsilon} f(z)^{-1 / v} .
$$

Sending $\epsilon$ to 0 , we have

$$
\liminf _{|y| \rightarrow \infty}|y|\left[|y|^{v} f(y)-1\right] \geq 0 .
$$

Thus, in view of (71),

$$
\lim _{|y| \rightarrow \infty}|y|\left[|y|^{\nu} f(y)-1\right]=0 .
$$

Let $e_{1}=(1,0, \ldots, 0), \ldots, e_{n}=(0, \ldots, 0,1)$. For any $x \in \mathbb{R}^{n}, 1 \leq i \leq n$, and $t \in \mathbb{R}$, let $y=y(x, t, i) \in \mathbb{R}^{n}$ be defined by

$$
t e_{i}=\frac{\lambda(x)^{2}(y-x)}{|y-x|^{2}} .
$$

Taking this $y$ in 70 and sending $t$ to 0 , we obtain, in view of (73),

$$
\frac{\partial f}{\partial x_{i}}(x)=\lim _{t \rightarrow 0} \frac{f\left(x+t e_{i}\right)-f(x)}{t}=-\frac{v x \cdot e_{i} f(x)}{\lambda(x)^{2}}=-v x_{i} f(x)^{1+2 / v} .
$$

By the continuity of $f$, we know that $f$ is in $C^{1}\left(\mathbb{R}^{n}\right)$, and we complete the proof of Lemma 5.8 by writing the above system of PDEs as $\frac{\partial}{\partial x_{i}}\left[f(x)^{-2 / v}-|x|^{2}\right]=0$ and solving it.

Acknowledgements. We thank W. Chen, C. Li and B. Ou for sending us their preprint [18] and thank B. Ou for an interesting talk on the work at a symposium. We are grateful to E. Lieb for the encouragement and for pointing out the need to study the (essential) uniqueness of solutions of (4) beyond the $L_{\mathrm{loc}}^{\infty}\left(\mathbb{R}^{n}\right)$ class and bringing to our attention solutions in [31] which are not maximizers. This has led us to study the regularity issue and to establish Theorems 1.2 and 1.3 We thank H. Brezis for pointing out the relation between Theorem 1.3 and Lemma A.1 in [7]. We thank L. Nirenberg for his interest in the work and for helping us to complete Appendix B (though not used in the present paper). The work was partially supported by NSF Grant DMS-0100819.

Added in proof. Question 2 is answered in the affirmative by Xingwang Xu in: A theorem on integral equations, preprint.

\section{References}

[1] Aleksandrov, A. D.: Uniqueness theorems for surfaces in the large. V. Vestnik Leningrad Univ. Mat. Mekh. Astronom. 13, 5-8 (1958); Amer. Math. Soc. Transl. 21, 412-416 (1962)

[2] Berestycki, H., Nirenberg, L.: On the method of moving planes and the sliding method. Bol. Soc. Brasil. Mat. (N.S.) 22, 1-37 (1991) Zbl 0784.35025 MR 93a:35048

[3] Branson, T.: Group representation arising from Lorentz conformal geometry. J. Funct. Anal. 74, 199-293 (1987) Zbl 0643.58036 MR 90b:22016

[4] Brezis , H., Cabré, X.: Some simple nonlinear PDE's without solutions. Boll. Un. Mat. Ital. 1, 223-262 (1998) Zbl 0907.35048 MR 99j:35001 
[5] Brezis, H., Dupaigne, L., Tesei, A.: On a semilinear elliptic equation with inverse-square potential. Preprint

[6] Brezis, H., Kato, T.: Remarks on the Schrödinger operator with singular complex potentials. J. Math. Pures Appl. 58, 137-151 (1979) Zbl 0408.35025 MR 80i:35135

[7] Brezis, H., Lieb, E.H.: Minimum action solutions of some vector field equations. Comm. Math. Phys. 96, 97-113 (1984) Zbl 0579.35025 MR 86d:35045

[8] Caffarelli, L., Gidas, B., Spruck, J.: Asymptotic symmetry and local behavior of semilinear equations with critical Sobolev growth. Comm. Pure Appl. Math. 42, 271-297 (1989) Zbl 0702.35085 MR 90c:35075

[9] Carlen, E., Loss, M.: Extremals of functionals with competing symmetries. J. Funct. Anal. 88, 437-456(1990) Zbl 0705.46016 MR 91f:42021

[10] Carlen, E., Loss, M.: Competing symmetries of some functionals arising in mathematical physics. In: Stochastic Processes, Physics and Geometry, S. Albeverio (ed.), World Sci., 277288 (1990) MR 92j:81338

[11] Carlen, E., Loss, M.: Competing symmetries, the logarithmic HLS inequality and Onofri's inequality on $\mathbb{S}^{n}$. Geom. Funct. Anal. 2, 90-104 (1990) Zbl 0754.47041 MR 93b:58170

[12] Carlen, E., Loss, M.: On the minimization of symmetric functionals. Rev. Math. Phys. 6, 1011-1032 (1994) Zbl 0871.47047 MR 96k:58068

[13] Chang, S. Y. A., Gursky, M., Yang, P.: An a priori estimate for a fully nonlinear equation on four-manifolds. J. Anal. Math. 87, 151-186 (2002) Zbl 01931226 MR 1945280

[14] Chang, S. Y. A., Gursky, M., Yang, P.: Entire solutions of a fully nonlinear equation. Preprint

[15] Chang, S. Y. A., Yang, P.: On uniqueness of solutions of $n$th order differential equations in conformal geometry. Math. Res. Lett. 4, 91-102 (1997) Zbl 0903.53027 MR 97m:58204

[16] Chang, S. Y. A., Yang, P.: Non-linear partial differential equations in conformal geometry. In: Proceedings of ICM 2002, Vol. I, Higher Ed. Press, Beijing, 189-207 (2002) MR 1989185

[17] Chen, W., Li, C.: Classification of solutions of some nonlinear elliptic equations. Duke Math. J. 63, 615-622 (1991) Zbl 0768.35025 MR 93e:35009

[18] Chen, W., Li, C., Ou, B.: Classification of solutions for an integral equation. Preprint.

[19] Fefferman, C., R. Graham, C.: Conformal invariants. In: Élie Cartan et les Mathématiques d'aujourd'hui, Astérisque 1985, 95-116 Zbl 0602.53007 MR 87g:53060

[20] Giaquinta, M., Giusti, E.: On the regularity of the minima of variational integrals. Acta Math. 148, 31-46 (1982) Zbl 0494.49031 MR 84b:58034

[21] Gidas, B., Ni, W. M., Nirenberg, L.: Symmetry and related properties via the maximum principle. Comm. Math. Phys. 68, 209-243 (1979) Zbl 0425.35020 MR 80h:35043

[22] Gidas, B., Ni, W. M., Nirenberg, L.: Symmetry of positive solutions of nonlinear elliptic equations in $\mathbb{R}^{n}$. In: Mathematical Analysis and Applications, Adv. in Math. Suppl. Stud. 7A, 369-402 (1981) Zbl 0469.35052 MR 84a:35083

[23] Gidas, B., Spruck, J.: Global and local behavior of positive solutions of nonlinear elliptic equations. Comm. Pure Appl. Math. 34, 525-598 (1981) Zbl 0465.35003 MR 83f:35045

[24] Li, A., Li, Y. Y.: On some conformally invariant fully nonlinear equations. Comm. Pure Appl. Math. 56, 1414-1464 (2003) MR 1988895

[25] Li, A., Li, Y. Y.: A general Liouville type theorem for some conformally invariant fully nonlinear equations. arXiv:math.AP/0301239 v1 21 Jan 2003

[26] Li, A., Li, Y. Y.: Further results on Liouville type theorems for some conformally invariant fully nonlinear equations. arXiv:math.AP/0301254 v1 22 Jan 2003

[27] Li, A., Li, Y. Y.: On some conformally invariant fully nonlinear equations. Part II: Liouville, Harnack and Yamabe. In preparation 
[28] Li. Y. Y.: Remark on some conformally invariant integral equations: the method of moving spheres. arXiv:math.AP/0307093 v1 8 Jul 2003; v2 18 Jul 2003

[29] Li, Y. Y., Zhang, L.: Liouville type theorems and Harnack type inequalities for semilinear elliptic equations. J. Anal. Math. 90, 27-87 (2003)

[30] Li, Y. Y., Zhu, M.: Uniqueness theorems through the method of moving spheres. Duke Math. J. 80, 383-417 (1995) Zbl 0846.35050 MR 96k:35061

[31] Lieb, E.: Sharp constants in the Hardy-Littlewood-Sobolev and related inequalities, Ann. of Math. 118, 349-374 (1983) Zbl 0527.42011 MR 86i:42010

[32] Lieb, E., Loss, M.: Analysis. 2nd ed., Amer. Math. Soc., Providence, RI (2001). Zbl 0966.26002 MR 2001i:00001

[33] Lin, C. S.: A classification of solutions of a conformally invariant fourth order equation in $\mathbb{R}^{n}$. Comment. Math. Helv. 73, 206-231 (1998) Zbl 0933.35057 MR 99c:35062

[34] Mazzeo, R., Pacard, F.: Constant scalar curvature metrics with isolated singularities. Duke Math. J. 99, 353-418 (1999) Zbl 0945.53024 MR 1712628

[35] McKenna, P. J., Reichel, W.: Radial solutions of singular nonlinear biharmonic equations and applications to conformal geometry. Electron. J. Differential Equations 2003, No. 37, 1-13. MR 1971023

[36] Ou, B.: A remark on a singular integral equation. Houston J. Math. 25, 181-184 (1999) Zbl 0965.45001 MR 2000e:45004

[37] Paneitz, S.: A quartic conformally covariant differential operator for arbitrary pseudoRiemannian manifolds. Preprint (1983)

[38] Serrin, J.: A symmetry problem in potential theory. Arch. Rat. Mech. Anal. 43, 304-318 (1971) Zbl 0222.31007 MR 48 \#11545

[39] Stein, E. M.: Singular Integrals and Differentiability Properties of Functions. Princeton Univ. Press, Princeton, NJ (1970) Zbl 0207.13501 MR 58 \#2467

[40] Viaclovsky, J.: Conformal geometry, contact geometry, and the calculus of variations. Duke Math. J. 101, 283-316 (2000) Zbl 0990.53035 MR 2001b:53038

[41] Viaclovsky, J.: Conformally invariant Monge-Ampère equations: global solutions. Trans. Amer. Math. Soc. 352, 4371-4379 (2000) Zbl 0951.35044 MR 2000m:35067

[42] Wei, J., Xu, X.: Classification of solutions of higher order conformally invariant equations. Math. Ann. 313, 207-228 (1999) Zbl 0940.35082 MR 2000a:58093

[43] $\mathrm{Xu}, \mathrm{X}$.: Uniqueness theorem for the entire positive solutions of biharmonic equations in $\mathbb{R}^{n}$. Proc. Roy. Soc. Edinburgh Sect. A 130, 651-70 (2000) Zbl 0961.35037 MR 2001f:35143

[44] Xu, X.: Exact solution of nonlinear conformally invariant integral equation in $\mathbb{R}^{3}$. Preprint 